\title{
Under-Foliage Object Imaging Using SAR Tomography and Polarimetric Spectral Estimators
}

\author{
Yue Huang, Laurent Ferro-Famil, Member, IEEE, and Andreas Reigber, Senior Member, IEEE
}

\begin{abstract}
This paper addresses the imaging of objects located under a forest cover using polarimetric synthetic aperture radar tomography (POLTOMSAR) at L-band. High-resolution spectral estimators, able to accurately discriminate multiple scattering centers in the vertical direction, are used to separate the response of objects and vehicles embedded in a volumetric background. A new polarimetric spectral analysis technique is introduced and is shown to improve the estimation accuracy of the vertical position of both artificial scatterers and natural environments. This approach provides optimal polarimetric features that may be used to further characterize the objects under analysis. The effectiveness of this novel technique for POLTOMSAR is demonstrated using fully polarimetric L-band airborne data sets acquired by the German Aerospace Center (DLR)'s E-SAR system over the test site in Dornstetten, Germany.
\end{abstract}

Index Terms-Multibaseline PolInSAR, SAR tomography, underfoliage imaging.

\section{GLOSSARY OF NOTATION}

$\mathbf{A}^{\mathrm{T}}$

$\mathbf{A}^{\dagger}$

$\operatorname{tr}(\mathbf{A})$

$\mathbf{A} \odot \mathbf{B}$

$\mathbf{A} \otimes \mathbf{B}$

$\|\mathbf{A}\|_{\mathbf{W}}^{2}=\operatorname{tr}\left(\mathbf{A} \mathbf{W} \mathbf{A}^{\dagger}\right)$

$\mathbf{I}_{(m \times m)}$

$\mathbf{1}_{(m \times n)}$

$\delta_{k, l}$

$\mathrm{E}()$

$\mathcal{N}(\mathbf{m}, \mathbf{\Gamma})$
Transpose of the matrix $\mathbf{A}$. Conjugate transpose of $\mathbf{A}$. Hadamard product of $\mathbf{A}, \mathbf{B} \in \mathbb{C}^{m \times n}$, defined by $[\mathbf{A} \odot \mathbf{B}]_{i j}=[\mathbf{A}]_{i j}[\mathbf{B}]_{i j}$. Kronecker product of $\mathbf{A} \in$ $\mathbb{C}^{m \times n}$ and $\mathbf{B} \in \mathbb{C}^{p \times q}$.

Weighted Frobenius norm of $\mathbf{A} \in \mathbb{C}^{m \times n}$.

$(m \times m)$ identity matrix.

$(m \times n)$ matrix of ones. if $k=l$ and equal to zero otherwise).

Expectation operator.

Complex Gaussian distribution with mean $\mathbf{m}$ and covariance matrix $\Gamma$. Trace of $\mathbf{A} \in \mathbb{C}^{m \times m}$.

Kronecker delta (equal to one

Manuscript received October 11, 2011; revised April 15, 2011 and August 16, 2011; accepted September 11, 2011. Date of publication November 18, 2011; date of current version May 16, 2012.

Y. Huang and L. Ferro-Famil are with the SAR Polarimetry, Holography, Interferometry and Radargrammetry Team, Institute of Electronics and Telecommunications of Rennes, University of Rennes 1, 35042 Rennes, France (e-mail: yue.huang@univ-rennes1.fr; Laurent.ferro-famil@univ-rennes1.fr).

A. Reigber is with the Microwaves and Radar Institute, German Aerospace Center (DLR), 82234 Wessling, Germany (e-mail: andreas.reigber@dlr.de).

Color versions of one or more of the figures in this paper are available online at http://ieeexplore.ieee.org.

Digital Object Identifier 10.1109/TGRS.2011.2171494

$$
\begin{array}{ll}
\hat{\boldsymbol{\theta}}=\arg \max _{\boldsymbol{\theta}} f(\boldsymbol{\theta}) & \begin{array}{l}
\text { Maximizing argument of } \\
f(\boldsymbol{\theta}) .
\end{array} \\
\hat{\boldsymbol{\theta}}=\arg \max _{\theta, \text { loc }} f(\theta) \in \mathbb{C}^{n \times 1} & \begin{array}{l}
\text { Values of } \theta \text { corresponding to } \\
\text { the } n \text { largest local maxima of } \\
f(\boldsymbol{\theta}) .
\end{array}
\end{array}
$$

\section{INTRODUCTION}

$\mathrm{S}_{\mathrm{T}} \mathrm{Y}$ YNTHETIC aperture radar (SAR) tomography is the extension of conventional 2-D SAR imaging to three dimensions and is achieved by the formation of an additional synthetic aperture in elevation. The coherent combination of images acquired from several parallel flight tracks using multibaseline SAR interferometric (InSAR) (MB-InSAR) techniques allows a direct localization of multiple scattering contributions within a resolution cell, leading to a refined analysis of volume structures, like forests or dense urban areas. Airborne SAR tomography was first demonstrated in [1] where tomograms for various polarization channels have been analyzed. The joint use of data sets acquired in a polarimetric MB-InSAR (MB-PolInSAR) configuration permits to both estimate 3-D distribution of volumetric structures and extract their physical features [2], [3].

Three-dimensional SAR focusing may be considered as a spectral estimation problem, and a wide variety of spectral analysis techniques can be used to perform tomography, ranging from classical Fourier-based methods to high-resolution (HR) approaches. It is well known that nonparametric approaches, like beamforming and Capon method, are, in general, more robust to focusing artifacts, whereas parametric approaches, like multiple signal classification (MUSIC) and maximum likelihood (ML) estimators, are characterized by a better vertical resolution. Capon's spectral estimation approach has been applied to the study of under-foliage objects, to extract the shape of objects and canopy profile [2]. Considering the complex structure of such objects, HR approaches are required to discriminate their closely spaced scattering features in the vertical direction. The performance of these spectral analysis techniques is conditioned by the statistical nature of the scattering response of the observed objects. As it has been shown in [4] and [5], under-foliage objects may be associated to a series of complex scattering centers with a deterministic response embedded within a speckle-affected environment.

In this case, usual spectral estimators may reach some limitations due to their lack of adaptation to the statistical features of the backscattered information. It has been shown in [6] and [7] that subspace methods such as weighted noise subspace 


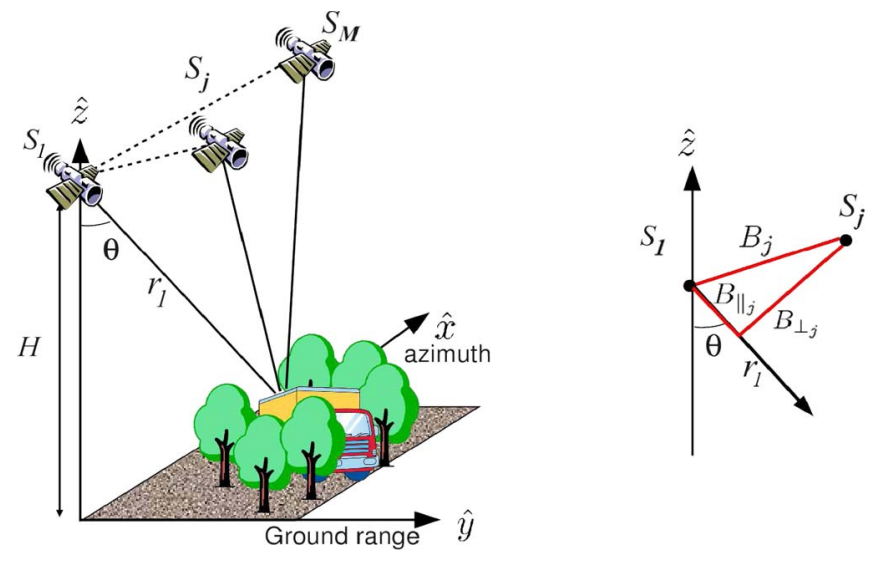

Fig. 1. MB-InSAR acquisition geometry for under-foliage object imaging.

fitting (NSF) and weighted signal subspace fitting (SSF) can cope with data showing complex statistical properties and can be applied to arbitrary array structure. In particular, the SSF estimator provides a prominent performance for direction-ofarrival estimation in the presence of highly correlated signals. In order to overcome limitations due to the unknown scattering nature of the imaged objects, we propose to apply weighted subspace fitting (WSF) estimators to tomographic focusing.

Considering polarimetric data, the NSF estimator has been implemented in the dual-polarization case, as shown in [8]. In order to further discriminate and characterize the objects under analysis using their polarimetric responses, we extend the NSF estimator to the fully polarimetric (FP) case, named FPNSF estimator. An analytic solution for the FP-NSF estimator optimization is derived that maintains the complexity of this technique to the one of the single-polarization (SP) case.

This paper is outlined as follows. In Section II, general SAR tomographic signal models are given under different cases, corresponding to coherent (deterministic), distributed, and hybrid scatterers that may be encountered when observing underfoliage objects. FP tomographic signal models for under-foliage object imaging are derived in Section III, and the proposed FP tomographic estimator is presented in Section IV. The performance of our approach is demonstrated using numerical examples in Section V and MB-PolInSAR data sets acquired by the German Aerospace Center (DLR)'s E-SAR system at L-band over Dornstetten in Section VI.

\section{SAR TOMOgRAPHiC Signal Models}

The MB-InSAR acquisition geometry for under-foliage object imaging is shown in Fig. 1 and consists of $M$ acquisition positions, each pair of which is separated by a baseline. The baseline distance between the master position and the $j$ th position, noted as $B_{j}$, can be decomposed into $B_{\|_{j}}$, a component parallel to the slant-range direction, and a perpendicular one $B_{\perp_{j}}$, aligned with the cross-range direction. Considering an azimuth-range resolution cell that contains $n_{s}$ backscattering contributions from scatterers located at different heights and assuming no decorrelation between the different acquisitions, the received data vector $\mathbf{y} \in \mathbb{C}^{M \times 1}$ can be formulated as follows:

$$
\mathbf{y}(l)=\sum_{i=1}^{n_{s}} s_{i} \mathbf{a}\left(z_{i}\right)+\mathbf{n}(l)=\mathbf{A}(\mathbf{z}) \mathbf{s}+\mathbf{n}(l)
$$

where $l=1, \ldots, L$ indicates one of the $L$ independent realizations of the signal acquisition, also called looks. The source signal vector $\mathbf{S}=\left[s_{1}, \ldots, s_{n_{s}}\right]^{\mathrm{T}}$ contains the unknown complex reflection coefficient of the $n_{s}$ scatterers, and $\mathbf{n} \in$ $\mathbb{C}^{M \times 1}$ represents the complex additive noise, assumed to be Gaussianly distributed with zero mean variance $\sigma_{n}^{2}$ and to be white in time and space with, i.e., $\mathbf{n} \sim \mathcal{N}\left(\mathbf{0}, \sigma_{n}^{2} \mathbf{I}_{(M \times M)}\right)$ and $\mathrm{E}\left(\mathbf{n}(l) \mathbf{n}^{\dagger}(k)\right)=\sigma_{n}^{2} \mathbf{I}_{(M \times M)} \delta_{l, k}$. The steering vector $\mathbf{a}(z)$ contains the interferometric phase information associated to a source located at the elevation position $z$ above the reference focusing plane and is given by

$$
\mathbf{a}(z)=\left[1, \exp \left(j k_{z_{2}} z\right), \ldots, \exp \left(j k_{z_{M}} z\right)\right]^{\mathrm{T}}
$$

where $k_{z_{j}}=(4 \pi / \lambda)\left(B_{\perp_{j}} / r_{1} \sin \theta\right)$ is the two-way vertical wavenumber between the master and the $j$ th acquisition tracks. The carrier wavelength is represented by $\lambda$, whereas $\theta$ stands for the incidence angle and $r_{1}$ is the slant-range distance between the master track and the scatterer. The steering matrix $\mathbf{A}(\mathbf{z})$ consists of $n_{s}$ steering vectors corresponding each to a backscattering source

$$
\mathbf{A}(\mathbf{z})=\left[\mathbf{a}\left(z_{1}\right), \ldots, \mathbf{a}\left(z_{n_{s}}\right)\right]
$$

with $\mathbf{z}=\left[z_{1}, \ldots, z_{n_{s}}\right]^{\mathrm{T}}$ being the vector of unknown source heights.

Considering now the interferometric decorrelation between different acquisitions, the initial model in (1) may be reformulated as a sum of contributions from random sources [9]

$$
\mathbf{y}(l)=\sum_{i=1}^{n_{s}} \mathbf{x}_{i}(l) \odot \mathbf{a}\left(z_{i}\right)+\mathbf{n}(l)
$$

where $\mathbf{x}_{i} \in \mathbb{C}^{M \times 1}$ accounts for both the reflection coefficient of the $i$ th source $s_{i}$ and its potential variations between the interferometric acquisitions or over the $L$ realizations.

The problem addressed in this paper concerns the estimation of the elevation $z_{i}$ and reflectivity $\sigma_{i}=\left|s_{i}\right|^{2}$ of each of the $n_{s}$ sources from independent realizations of the noisy MB-InSAR measured signal $\mathbf{y}(l), l=1, \ldots, L$. Depending on the type of scatterer under observation, the source signal $\mathbf{x}_{i}$ possesses varying statistical properties. The resulting composite signal $\mathbf{y}(l)$ may then follow different scattering behaviors that are detailed in the following.

\section{A. Distributed Scatterer Model}

Distributed media are characterized by a scattering response having a random behavior conferred by the speckle effect. Under some assumptions summarized in [10] and [11], the scattered signal may be modeled using a multiplicative random term following a centered complex Gaussian distribution with unitary variance. Considering the $n_{s}$ sources as distributed 
scatterers with speckle-affected responses, each source signal can be expressed as

$$
\mathbf{x}_{i}(l)=s_{i} \mathbf{x}_{u_{i}}(l) \in \mathbb{C}^{M \times 1}, \quad \text { with } \mathbf{x}_{u_{i}}(l) \sim \mathcal{N}\left(\mathbf{0}, \mathbf{C}_{i}\right)
$$

where $\mathbf{x}_{u_{i}}(l)$ represents the multiplicative speckle term and $s_{i}$ is the complex response of the $i$ th scatterer, its reflectivity being given by $\sigma_{i}=\left|s_{i}\right|^{2}$. The stochastic signals $\left\{\mathbf{x}_{u_{i}}(l)\right\}_{l=1}^{L}$ are independent and identically distributed, i.e., $\mathrm{E}\left(\mathbf{x}_{u_{i}}(l) \mathbf{x}_{u_{i}}^{\dagger}(k)\right)=$ $\mathbf{C}_{i} \delta_{l, k}$. The $(M \times M)$ covariance matrix $\mathbf{C}_{i}$ describes the interferometric coherence and, for a well-calibrated acquisition system, contains unitary diagonal elements, whereas offdiagonal terms depend on the acquisition conditions.

This kind of source signal is well adapted to the modeling of scattering over natural environments, like rough surfaces, ground, and volumetric environments. Under this unconditional model (UM) assumption [4], the received data vector in (5) is named $\mathbf{y}_{u}(l)$ and follows a centered Gaussian random distribution, i.e., $\mathbf{y}_{u}(l) \sim \mathcal{N}(\mathbf{0}, \mathbf{R})$, and its covariance matrix is given by

$$
\mathbf{R}=\sum_{i=1}^{n_{s}} \sigma_{i} \mathbf{C}_{i} \odot \mathbf{a}\left(z_{i}\right) \mathbf{a}^{\dagger}\left(z_{i}\right)+\sigma_{n}^{2} \mathbf{I}_{(M \times M)} .
$$

The reflectivity $\sigma_{i}$ and height $z_{i}$ of each scatterer are the unknown parameters to be estimated from $\mathbf{R}$.

\section{B. Deterministic Scatterer Model}

In the case of deterministic scatterers, the backscattered source signal is frozen over all the observations and presents no decorrelation between the different acquisitions. For a wellcalibrated system, its form is given by

$$
\mathbf{x}_{i}(l)=s_{i} \mathbf{x}_{c_{i}}(l) \in \mathbb{C}^{M \times 1}, \quad \text { with } \mathbf{x}_{c_{i}}(l)=\mathbf{1}_{(M \times 1)} .
$$

This behavior is generally related to specular scattering mechanisms and can be observed over coherent scatterers like calibrators, facets facing the radar, and double-bounce reflections over dihedral-like objects having smooth surfaces (like ground-trunk interactions and double-bounce reflections between an object and the ground), or may be linked to resonant behaviors over quasi-periodic media [12].

Under this conditional model (CM) assumption [4], the received data vector in (7) is named $\mathbf{y}_{c}(l)$ and follows a Gaussian distribution with mean $\mathbf{A}(\mathbf{z}) \mathbf{s}$ and covariance matrix equal to the additive noise one, i.e., $\mathbf{y}_{c}(l) \sim \mathcal{N}\left(\mathbf{A}(\mathbf{z}) \mathbf{s}, \sigma_{n}^{2} \mathbf{I}_{(M \times M)}\right)$. The equivalent to the matrix $\mathbf{R}$ given in (6) is

$$
\mathbf{R}=\mathrm{E}\left(\mathbf{y}_{c}(l) \mathbf{y}_{c}^{\dagger}(l)\right)=\mathbf{A}(\mathbf{z}) \mathbf{s s}^{\dagger} \mathbf{A}^{\dagger}(\mathbf{z})+\sigma_{n}^{2} \mathbf{I}_{(M \times M)}
$$

and may be used to estimate the reflectivity $\sigma_{i}$ and height $z_{i}$ of each source.

\section{Hybrid Scatterer Model}

SAR scenes generally consist of a mixture of coherent and distributed scatterers [13]. In such cases, a hybrid MB-InSAR signal model, introduced by Sauer et al. [14], [15], may be used to describe the received signal, as it includes both deterministic and distributed contributions

$$
\begin{aligned}
\mathbf{y}(l) & =\mathbf{y}_{c}(l)+\mathbf{y}_{u}(l) \\
& =\sum_{i=1}^{n_{s_{1}}} s_{i} \mathbf{x}_{c_{i}}(l) \odot \mathbf{a}\left(z_{i}\right)+\sum_{i=1}^{n_{s_{2}}} s_{i} \mathbf{x}_{u_{i}}(l) \odot \mathbf{a}\left(z_{i}\right)+\mathbf{n}(l) .
\end{aligned}
$$

This type of signals can be frequently encountered when dealing with intermediate-resolution SAR images. In this paper, this hybrid model is used to describe the signal received from objects embedded in forests, since it contains deterministic CM components, due to double-bounce mechanisms or to coherent scatterers that are part of the object, and distributed UM terms related to scattering over the ground or over the forest canopy. The reflectivity $\sigma_{i}$ and height $z_{i}$ of each source may be estimated from $\mathbf{R}=\mathrm{E}\left(\mathbf{y}(l) \mathbf{y}^{\dagger}(l)\right)$ which is characterized by the following form:

$$
\mathbf{R}=\mathbf{A}(\mathbf{z}) \mathbf{P} \mathbf{A}^{\dagger}(\mathbf{z})+\sigma_{n}^{2} \mathbf{I}_{(M \times M)}
$$

where $\mathbf{P}$ represents the $\left(n_{s} \times n_{s}\right)$ source signal covariance matrix and $n_{s}$ is the number of effective sources.

In order to illustrate the statistical properties of such a signal, one may consider a two-component hybrid source signal represented by $\mathbf{x}(l)=\sqrt{\sigma_{u}} \mathbf{x}_{u}(l)+\sqrt{\sigma_{c}} \mathbf{x}_{c}(l) \in \mathbb{C}^{2 \times 1}$, where the UM and CM terms have been defined in (5) and (7), respectively. The covariance matrix of the UM contribution is then $\mathbf{R}_{u}=\sigma_{u} \mathbf{I}_{2 \times 2}$, whereas the CM term has a covariance matrix given by $\mathbf{R}_{c}=\sigma_{c} \mathbf{1}_{2 \times 2}$. Therefore, the covariance matrix of the global hybrid signal $\mathbf{x}$ may be expressed as

$$
\mathbf{R}_{\mathbf{x}}=\mathbf{R}_{u}+\mathbf{R}_{c}=\left(\sigma_{u}+\sigma_{c}\right)\left[\begin{array}{cc}
1 & \rho \\
\rho^{*} & 1
\end{array}\right]
$$

where the correlation between the sources is defined as

$$
\rho=\frac{1}{1+U C R}, \quad \text { with } U C R=\frac{\sigma_{u}}{\sigma_{c}}
$$

where $U C R$ represents the ratio of $\mathrm{UM}$ to $\mathrm{CM}$ scattered power. If the coherent contribution of the hybrid signal dominates, i.e., $\rho \rightarrow 1$ and $U C R \rightarrow 0$, then $\mathbf{R}_{\mathrm{x}}$ becomes singular, making difficult the parameter estimation by spectral techniques that cannot cope with highly correlated signals, like MUSIC [16], [17]. In the opposite case, when $U C R \gg 0$, the uncorrelated contribution predominates, and hybrid scatterers tend to be behave like distributed ones.

\section{POLTOMSAR SignAL MOdEL}

The polarimetric response of a scatterer is fully described by its $(2 \times 2)$ complex scattering matrix $\mathbf{S}$ given by

$$
\mathbf{S}=\left[\begin{array}{ll}
S_{h h} & S_{v h} \\
S_{h v} & S_{v v}
\end{array}\right] .
$$

This paper considers data acquired in a monostatic configuration, for which the matrix $\mathbf{S}$ is symmetric, i.e., $S_{h v}=S_{v h}$. The scattering matrix can be vectorized using, for instance, the 
Pauli basis matrix set $\left\{\boldsymbol{\Psi}_{p}\right\}_{p=1, \ldots, 3}[18]$, in order to build a target vector $\mathbf{v}$

$$
\mathbf{v}=\frac{1}{\sqrt{2}}\left[S_{h h}+S_{v v}, S_{h h}-S_{v v}, 2 S_{h v}\right]^{\mathrm{T}}=s \mathbf{k}
$$

where $\sigma=|s|^{2}=\mathbf{V}^{\dagger} \mathbf{v}$ represents the polarimetric span [18] of the scatterer response and $\mathbf{k}=\left[k_{1}, k_{2}, k_{3}\right]^{\mathrm{T}} \in \mathbb{C}^{3 \times 1}$ represents a unitary polarimetric target vector, i.e., $\mathbf{k}^{\dagger} \mathbf{k}=1$. In an MBPolInSAR configuration, the array response may be represented by rearranging the $M$ acquired polarimetric signals $\mathbf{v}_{j}$, with $j=1, \ldots, M$ being the track index, under the form of a $3 M$ element vector $\mathbf{y}_{P}$ composed of three MB-InSAR components, each related to a polarization channel

$$
\mathbf{y}_{P}=\left[\mathbf{y}_{1}^{\mathrm{T}} \mathbf{y}_{2}^{\mathrm{T}}, \mathbf{y}_{3}^{\mathrm{T}}\right]^{\mathrm{T}} \in \mathbb{C}^{3 M \times 1}
$$

where $\mathbf{y}_{p} \in \mathbb{C}^{3 M \times 1}$, with $p=1,2$, or 3 , represents the MBInSAR response for the $p$ th polarimetric channel, i.e., $\left[\mathbf{y}_{p}\right]_{j}=$ $\left[\mathbf{v}_{j}\right]_{p}$. Using this convention of representation, the polarimetric steering vector and steering matrix are given by

$$
\begin{aligned}
& \mathbf{a}\left(z_{i}, \mathbf{k}_{i}\right)=\mathbf{k}_{i} \otimes \mathbf{a}\left(z_{i}\right) \\
& \mathbf{A}(\mathbf{z}, \mathbf{K})=\left[\mathbf{a}\left(z_{1}, \mathbf{k}_{1}\right), \ldots, \mathbf{a}\left(z_{n_{s}}, \mathbf{k}_{n_{s}}\right)\right]
\end{aligned}
$$

where $\mathbf{k}_{i}$ is the polarimetric target vector of the $i$ th source and $\mathbf{K}=\left[\mathbf{k}_{1}, \ldots, \mathbf{k}_{n_{s}}\right]$. A polarimetric steering vector can be defined using five real coefficients given by the elevation $z$ and the real and imaginary parts of two complex numbers defining a unitary three-element polarimetric complex vector whose absolute phase is arbitrary. Similarly to the SP expression given in (1), the received MB-PolInSAR signal $\mathbf{y}_{P}(l)$ may be formulated as

$$
\mathbf{y}_{P}(l)=\mathbf{A}(\mathbf{z}, \mathbf{K}) \mathbf{s}(l)+\mathbf{n}(l) \in \mathbb{C}^{3 M \times 1}
$$

where, similarly to the SP case, $\mathbf{s}(l) \in \mathbb{C}^{n_{s} \times 1}$ represents a realization of the complex amplitude of the $i$ th source. Diverse model assumptions, corresponding to UM, CM, and hybrid signals, given for SP signals, can be similarly used for POLTOMSAR signals. The reflectivity $\sigma_{i}$, scattering vector $\mathbf{k}_{i}$, and height $z_{i}$ of each source may be estimated from $\mathbf{R}_{P}=$ $\mathrm{E}\left(\mathbf{y}_{P}(l) \mathbf{y}_{P}^{\dagger}(l)\right)$, given by

$$
\mathbf{R}_{P}=\mathbf{A}(\mathbf{z}, \mathbf{K}) \mathbf{P A}^{\dagger}(\mathbf{z}, \mathbf{K})+\sigma_{n}^{2} \mathbf{I}_{(3 M \times 3 M)} .
$$

\section{SAR TOMOGRAPHIC RECONSTRUCTION}

\section{A. Estimation of MB-PolInSAR Signals}

As it has been mentioned previously, source characteristics can be estimated from the covariance matrix of the received signal $\mathbf{R}_{P}=\mathrm{E}\left(\mathbf{y}_{P} \mathbf{y}_{P}^{\dagger}\right)$. However, in practice, this information needs to be estimated. For a locally Gaussian statistical behavior, an ML estimate of $\mathbf{R}_{P}$ may be computed from $L$ independent locations surrounding the pixel under analysis as

$$
\hat{\mathbf{R}}_{P}=\frac{1}{L} \sum_{l=1}^{L} \mathbf{y}_{P}(l) \mathbf{y}_{P}^{\dagger}(l)
$$

Under the hypothesis of a uniform true reflectivity and for a sufficient number of looks $L, \hat{\mathbf{R}}_{P}$ may be used instead of $\mathbf{R}_{P}$ to perform tomography. Other techniques aiming to estimate the covariance matrix information for nonuniform reflectivity textures are given in [19] and [20].

The number of sources $n_{s}$ is, in general, unknown and needs to be estimated from the measured data. Some commonly used model order (MO) selection (MOS) techniques based on statistical approaches, e.g., information theoretic criterion (ITC), minimum description length, and Akaike information criterion [21]-[23], may be used to determine $\hat{n}_{s}$. Other modern methods, which are not based on a specific probability density function of the noise but rather on subspace orthogonality and shift-invariance properties, can also give accuracy estimates, as shown in [24] and [25].

\section{B. SP Tomography}

Spectral estimation techniques may be applied to perform SP tomography, by estimating the height profile of a medium and the corresponding set of reflectivity coefficients, using MBInSAR data sets acquired for a given polarization channel. In the following are presented some classical monodimensional spectral estimators, like beamforming, Capon method, and MUSIC, and multidimensional ones based on WSF-based approaches, like SSF and NSF.

1) Classical Monodimensional Techniques: These approaches determine $\hat{\mathbf{z}}$, an estimate of the elevation of the scatterers under observation, as the coordinates of the $\hat{n}_{s}$ largest local maxima of an objective function with a scalar elevation argument $P(z)$

$$
\hat{\mathbf{z}}=\arg \max _{z, \text { loc }} P(z) .
$$

For the classical beamformer and Capon spectral estimation techniques, the objective function is given by the continuous estimate of the reflectivity $P_{B, C}(z)=\hat{\sigma}_{B, C}(z)$ defined as

$$
\begin{aligned}
\hat{\sigma}_{B}(z) & =\frac{\mathbf{a}^{\dagger}(z) \hat{\mathbf{R}} \mathbf{a}(z)}{M^{2}} \\
\hat{\sigma}_{C}(z) & =\frac{1}{\mathbf{a}^{\dagger}(z) \hat{\mathbf{R}}^{-1} \mathbf{a}(z)} .
\end{aligned}
$$

Once the set of scatterer elevations $\hat{\mathbf{z}}$ is estimated using (20), the corresponding reflectivities can be obtained from (21). The selection of discrete sources from peaks of the reflectivity spectrum confers to the beamformer and Capon estimation techniques an important sensitivity to the acquisition configuration and, particularly, to the presence of spurious sidelobes related to an irregular baseline sampling. The beamformer is known to show a low resolution and may then overlook some closely spaced scatterers, whereas Capon's technique possesses an improved resolution but a reduced radiometric accuracy.

MUSIC is a subspace-based single-dimension technique, whose objective function is a measure of the orthogonality 
between a steering vector and the noise subspace and is given by

$$
P_{M}(z)=\frac{1}{\mathbf{a}^{\dagger}(z) \hat{\mathbf{E}}_{n} \hat{\mathbf{E}}_{n}^{\dagger} \mathbf{a}(z)}
$$

where $\hat{\mathbf{E}}_{n}$ represents an estimate of the noise subspace and is described in the next section. Once $\hat{\mathbf{z}}$ is determined by inserting (22) in (20), a least squares (LS) estimate of the complex reflectivity vector $\hat{\mathbf{s}}(l)$ can be obtained from [9], [26]

$$
\begin{aligned}
\hat{\mathbf{s}}(l) & =\arg \min _{\mathbf{s}}\|\mathbf{y}(l)-\mathbf{A}(\hat{\mathbf{z}}) \mathbf{s}\|^{2} \\
& =\left(\mathbf{A}^{\dagger}(\hat{\mathbf{z}}) \mathbf{A}(\hat{\mathbf{z}})\right)^{-1} \mathbf{A}^{\dagger}(\hat{\mathbf{z}}) \mathbf{y}(l)
\end{aligned}
$$

from which one may compute an $L$-look estimate of the reflectivity of each source using

$$
\hat{\sigma}_{i}=\frac{1}{L} \sum_{l=1}^{L}\left|\hat{s}_{i}(l)\right|^{2} .
$$

Nonparametric approaches, like beamforming and Capon method, are generally used to globally appreciate the structure of a volumetric medium and the main trends of the continuous reflectivity distribution in elevation. For the analysis of discrete spectral components, they may fail to discriminate closely spaced scatterers due either to their limited resolution or to the presence of sidelobes that may induce an erroneous estimation of the source location. MUSIC generally presents better performances for the analysis of discrete sources, related to a better resolution. Nevertheless, like all parametric methods, MUSIC is sensitive to data modeling errors and, particularly, to those related to the estimated number of sources $\hat{n}_{s}$. Moreover, MUSIC is known to work well in the case of uncorrelated scatterers, i.e., $\rho=0$ in (11), but its performance may degrade significantly in the presence of correlated scatterers since the source signal covariance matrix tends to be singular. One of the main advantages of such techniques resides in the low numerical complexity of the monodimensional optimization described in (20).

2) Multidimensional WSF: Efficient spectral estimation techniques have been developed in [7] and [27], based on the eigendecomposition of the covariance matrix of the received MB-InSAR signal

$$
\mathbf{R}=\mathbf{A}(\mathbf{z}) \mathbf{P A}^{\dagger}(\mathbf{z})+\sigma_{n}^{2} \mathbf{I}_{(M \times M)}=\sum_{j=1}^{M} \lambda_{j} \mathbf{e}_{j} \mathbf{e}_{j}^{\dagger}
$$

where $\lambda_{1}, \ldots, \lambda_{M}$ denote the eigenvalues of $\mathbf{R}$ in nonincreasing order and $\mathbf{e}_{1}, \ldots, \mathbf{e}_{M}$ are the corresponding orthonormal eigenvectors. This may be rewritten as

$$
\mathbf{R}=\mathbf{E}_{s} \boldsymbol{\Lambda}_{s} \mathbf{E}_{s}^{\dagger}+\sigma_{n}^{2} \mathbf{E}_{n} \mathbf{E}_{n}^{\dagger}
$$

with $\boldsymbol{\Lambda}_{s}=\operatorname{diag}\left(\left[\lambda_{1}, \ldots, \lambda_{n_{s}}\right]\right), \mathbf{E}_{s}=\left[\mathbf{e}_{1}, \ldots, \mathbf{e}_{n_{s}}\right]$, and $\mathbf{E}_{n}=$ $\left[\mathbf{e}_{n_{s}+1}, \ldots, \mathbf{e}_{M}\right]$. It is clear from (25) that, if the signal covariance matrix $\mathbf{P}$ has rank $n_{s}$, then $\mathbf{E}_{n}$ is orthogonal to $\mathbf{A}(\mathbf{z}) \mathbf{P A}^{\dagger}(\mathbf{z})$, implying that the range space of $\mathbf{E}_{s}$ coincides with that of $\mathbf{A}(\mathbf{z})$. These relationships may be summarized using two simple relations

$$
\begin{array}{r}
\mathbf{E}_{s}-\mathbf{A}(\mathbf{z}) \mathbf{T}=\mathbf{0} \\
\mathbf{E}_{n}^{\dagger} \mathbf{A}(\mathbf{z})=\mathbf{0}
\end{array}
$$

where $\mathbf{T}$ is a full-rank $\left(n_{s} \times n_{s}\right)$ matrix. The matrix $\mathbf{E}_{s} \in$ $\mathbb{C}^{M \times n_{s}}$ is said to represent the signal subspace of $\mathbf{R}$, whereas $\mathbf{E}_{n} \in \mathbb{C}^{M \times\left(M-n_{s}\right)}$ is associated to its noise subspace.

In practice, the number of sources $n_{s}$ and the eigenstructure matrices need to be estimated from the sample covariance matrix

$$
\hat{\mathbf{R}}=\hat{\mathbf{E}}_{s} \hat{\boldsymbol{\Lambda}}_{s} \hat{\mathbf{E}}_{s}^{\dagger}+\hat{\mathbf{E}}_{n} \hat{\boldsymbol{\Lambda}}_{n} \hat{\mathbf{E}}_{n}^{\dagger}
$$

Such estimates are consistent, but due to the intrinsic variability of $L$-look representations, there exists, in general, no value of $\mathbf{z}$ such that the relations between spaces specified in (27) are verified. Instead, one may determine the set of scatterer elevations $\hat{\mathbf{z}}$ that minimizes the left-hand side expressions (27) in the weighted LS sense, i.e., that optimizes a WSF criterion. Such an approach leads to the definition of two cost functions with vector argument. The weighted NSF cost function is defined as

$$
Q_{\mathrm{NSF}}(\mathbf{z})=\left\|\hat{\mathbf{E}}_{n}^{\dagger} \mathbf{A}(\mathbf{z})\right\|_{\mathbf{W}}^{2}=\operatorname{tr}\left(\mathbf{A}^{\dagger}(\mathbf{z}) \hat{\mathbf{E}}_{n} \hat{\mathbf{E}}_{n}^{\dagger} \mathbf{A}(\mathbf{z}) \mathbf{W}\right)
$$

whereas the weighted SSF cost function is given by

$$
Q_{\mathrm{SSF}}(\mathbf{z})=\left\|\hat{\mathbf{E}}_{s}-\mathbf{A}(\mathbf{z}) \mathbf{T}\right\|_{\mathbf{W}}^{2}=\operatorname{tr}\left(\mathbf{P}_{\mathbf{A}}^{\perp}(\mathbf{z}) \hat{\mathbf{E}}_{s} \mathbf{W} \hat{\mathbf{E}}_{s}^{\dagger}\right)
$$

where the fitting matrix $\mathbf{T}$ is replaced by it LS estimate $\hat{\mathbf{T}}=$ $\left(\mathbf{A}^{\dagger}(\mathbf{z}) \mathbf{A}(\mathbf{z})\right)^{-1} \mathbf{A}^{\dagger}(\mathbf{z}) \hat{\mathbf{E}}_{s}$ and

$$
\mathbf{P}_{\mathbf{A}}^{\perp}(\mathbf{z})=\left(\mathbf{I}_{(M \times M)}-\mathbf{A}(\mathbf{z})\left(\mathbf{A}^{\dagger}(\mathbf{z}) \mathbf{A}(\mathbf{z})\right)^{-1} \mathbf{A}^{\dagger}(\mathbf{z})\right)
$$

represents the orthogonal projector on the null space of $\mathbf{A}^{\dagger}(\mathbf{z})$. Both cost functions may be used to estimate the elevation of the scatterers using an $\hat{n}_{s}$-dimensional minimization

$$
\hat{\mathbf{z}}_{\mathrm{WSF}}=\arg \max _{\mathbf{z}} Q_{\mathrm{WSF}}(\mathbf{z})
$$

where the suffix WSF indicates one of the methods, NSF or SSF, mentioned previously. The reflectivity of each source may then be estimated by inserting the heights determined from (32) in the LS expression given in (23). It has been shown in [7] that any Hermitian positive semidefinite weighting matrix $\mathbf{W}$ yields consistent parameter estimates. In particular, a consistent estimate of $\mathbf{W}$ permits to obtain minimum variance estimates, which asymptotically reach the Cramér-Rao lower bound [27]. Such a value is given for each case in [7] and [6] as

$$
\begin{aligned}
\mathbf{W}_{\mathrm{SSF}_{\mathrm{opt}}} & =\left(\hat{\boldsymbol{\Lambda}}_{s}-\hat{\sigma}_{n}^{2} \mathbf{I}_{\left(\hat{n}_{s} \times \hat{n}_{s}\right)}\right)^{2} \hat{\boldsymbol{\Lambda}}_{s}^{-1} \\
\mathbf{W}_{\mathrm{NSF}_{\mathrm{opt}}} & =\left(\mathbf{A}^{\dagger}(\mathbf{z}) \hat{\mathbf{E}}_{s} \mathbf{W}_{\mathrm{SSF}_{\mathrm{opt}}}^{-1} \hat{\mathbf{E}}_{s}^{\dagger} \mathbf{A}(\mathbf{z})\right)^{-1} .
\end{aligned}
$$

Tomographic focusing using WSF techniques permits to reach a very high vertical resolution. For specific values of the 
weighting matrix $\mathbf{W}$, the WSF approaches can be asymptotically associated to other spectral estimators, like a multidimensional version of MUSIC [16] or ML methods under the CM and UM assumptions [4], used in [5] and [28] for the analysis of urban areas from MB-InSAR data sets.

The adaptation to the signal characteristics provided by the optimal $\mathbf{W}$ values given in (33) permits WSF-based tomographic techniques to discriminate closely spaced scatterers and accurately estimate their characteristics, independently of their statistical behavior, that may be related to the UM, CM, and hybrid models introduced previously. Coupling such an adaptive estimation, together with a multidimensional optimization, provides a lower sensitivity to focusing artifacts, like sidelobes, or to inaccurate estimates of the number of sources $\hat{n}_{s}$. The multidimensional subspace method, NSF, achieves a precise estimation for partially correlated scatterers but may reach some limitations for highly correlated ones, i.e., as $\rho \rightarrow 1$ in (11), whereas SSF outperforms the other methods for relevantly localizing highly correlated scatterers.

\section{FP Tomography}

FP tomography, which aims to estimate the height, reflectivity, and polarimetric scattering mechanism of each of the observed sources, possesses an improved potential, compared to SP 3-D focusing, for recovering some of the physical features of the observed medium. The coherent processing of MBPolInSAR is also expected to provide enhanced discrimination, due to the adaptation of the imaging approach to the electromagnetic features of the scatterers.

1) Conventional FP Estimators: The elevation and scattering mechanisms of the different scatterers are estimated as the coordinates of the $\hat{n}_{s}$ largest local maxima of a polarimetric objective function $P(z, \mathbf{k})$

$$
\hat{\mathbf{z}}, \hat{\mathbf{K}}=\arg \max _{z, \mathbf{k}, \text { loc }} P(z, \mathbf{k}) .
$$

Similarly to the SP case, the beamformer and Capon objective functions are given by continuous estimates of the reflectivity $P_{B, C}(z, \mathbf{k})=\hat{\sigma}_{B, C}(z, \mathbf{k})$, with

$$
\begin{aligned}
\hat{\sigma}_{B}(z, \mathbf{k}) & =\frac{\mathbf{a}^{\dagger}(z, \mathbf{k}) \hat{\mathbf{R}}_{P} \mathbf{a}(z, \mathbf{k})}{M^{2}} \\
\hat{\sigma}_{C}(z, \mathbf{k}) & =\frac{1}{\mathbf{a}^{\dagger}(z, \mathbf{k}) \hat{\mathbf{R}}_{P}^{-1} \mathbf{a}(z, \mathbf{k})} .
\end{aligned}
$$

The direct local maximization of the FP objective function in (34) would require searching solutions in a 5-D argument space. Rewriting the polarimetric steering vector as

$$
\mathbf{a}\left(z_{i}, \mathbf{k}_{i}\right)=\left(\mathbf{I}_{(3 \times 3)} \otimes \mathbf{a}\left(z_{i}\right)\right) \mathbf{k}_{i}=\mathbf{B}_{\mathbf{a}}\left(z_{i}\right) \mathbf{k}_{i}
$$

permits to formulate the estimators and reduce the search dimension to one

$$
P_{B}(z)=\max _{\mathbf{k}} P_{B}(z, \mathbf{k})=\frac{\lambda_{\max }\left(\mathbf{B}_{\mathbf{a}}^{\dagger}(z) \hat{\mathbf{R}}_{P} \mathbf{B}_{\mathbf{a}}(z)\right)}{M^{2}}
$$

$$
P_{C}(z)=\max _{\mathbf{k}} P_{C}(z, \mathbf{k})=\frac{1}{\lambda_{\min }\left(\mathbf{B}_{\mathbf{a}}^{\dagger}(z) \hat{\mathbf{R}}_{P}^{-1} \mathbf{B}_{\mathbf{a}}(z)\right)}
$$

where $\lambda_{\min }(\mathbf{M})$ and $\lambda_{\max }(\mathbf{M})$ represent the minimum and maximum eigenvalues of the positive semidefinite matrix $\mathbf{M}$, respectively. Once the $\hat{n}_{s}$ elevations are determined from the local maxima of (37), the corresponding scattering mechanisms can be estimated from

$$
\begin{aligned}
\hat{\mathbf{k}}_{B_{i}} & =\mathbf{e}_{\max }\left(\mathbf{B}_{\mathbf{a}}^{\dagger}\left(\hat{z}_{i}\right) \hat{\mathbf{R}}_{P} \mathbf{B}_{\mathbf{a}}\left(\hat{z}_{i}\right)\right) \\
\hat{\mathbf{k}}_{C_{i}} & =\mathbf{e}_{\min }\left(\mathbf{B}_{\mathbf{a}}^{\dagger}\left(\hat{z}_{i}\right) \hat{\mathbf{R}}_{P}^{-1} \mathbf{B}_{\mathbf{a}}\left(\hat{z}_{i}\right)\right)
\end{aligned}
$$

where $\mathbf{e}_{\min }(\mathbf{M})$ and $\mathbf{e}_{\max }(\mathbf{M})$ indicate the eigenvectors of $\mathbf{M}$ associated with $\lambda_{\min }(\mathbf{M})$ and $\lambda_{\max }(\mathbf{M})$, respectively. A similar approach may be applied to concentrate the FP MUSIC objective function

$$
\begin{aligned}
P_{M}(z) & =\max _{\mathbf{k}} P_{M}(z, \mathbf{k})=\frac{1}{\lambda_{\min }\left(\mathbf{B}_{\mathbf{a}}^{\dagger}(z) \hat{\mathbf{E}}_{n} \hat{\mathbf{E}}_{n}^{\dagger} \mathbf{B}_{\mathbf{a}}(z)\right)} \\
\hat{\mathbf{k}}_{M_{i}} & =\mathbf{e}_{\min }\left(\mathbf{B}_{\mathbf{a}}^{\dagger}\left(\hat{z}_{i}\right) \hat{\mathbf{E}}_{n} \hat{\mathbf{E}}_{n}^{\dagger} \mathbf{B}_{\mathbf{a}}\left(\hat{z}_{i}\right)\right)
\end{aligned}
$$

where $\hat{\mathbf{E}}_{n}$ represents here the eigenvectors of $\hat{\mathbf{R}}_{P}$ spanning its noise space. The reflectivity of the sources can be estimated, using an $L$-look polarimetric LS approach, as $\hat{\mathbf{s}}=\left(\mathbf{A}^{\dagger}(\hat{\mathbf{z}}, \hat{\mathbf{K}}) \mathbf{A}(\hat{\mathbf{z}}, \hat{\mathbf{K}})\right)^{-1} \mathbf{A}^{\dagger}(\hat{\mathbf{z}}, \hat{\mathbf{K}}) \mathbf{y}_{P}(l)$ and $\hat{\sigma}_{i}=(1 / L)$ $\sum_{l=1}^{L}\left|\hat{s}_{i}(l)\right|^{2}$.

These conventional estimators are considered as monodimensional, since the estimation of the elevation and the scattering mechanism is jointly realized by eigendecomposition. Such methods are computationally efficient but may reach some of the limitations mentioned in the SP case.

2) FP-NSF Estimator: In this section, we propose an FP version of the multidimensional and model-adaptive NSF spectral estimator introduced in [5]. This derivation is an extension to three polarization channels of the efficient approach proposed in [8] for the dual-polarization case. The objective of the FP-NSF approach is to determine $\hat{\mathbf{z}}$ and $\hat{\mathbf{K}}$ that minimize the NSF weighted cost function given by

$$
Q_{\mathrm{NSF}}(\mathbf{z}, \mathbf{K})=\operatorname{tr}\left(\mathbf{A}^{\dagger}(\mathbf{z}, \mathbf{K}) \hat{\mathbf{E}}_{n} \hat{\mathbf{E}}_{n}^{\dagger} \mathbf{A}(\mathbf{z}, \mathbf{K}) \mathbf{W}\right) .
$$

Note that any symmetric positive definite $\mathbf{W}$ will yield consistent parameter estimates [8]. The optimal weighting matrix that makes the estimation error covariance attain its Cramér-Rao low bound [8], [27] is given by

$$
\mathbf{W}_{\mathrm{opt}}=\left(\mathbf{A}^{\dagger}(\mathbf{z}, \mathbf{K}) \hat{\mathbf{E}}_{s}\left(\hat{\boldsymbol{\Lambda}}_{s}-\sigma_{n}^{2} \mathbf{I}_{\left(\hat{n}_{s} \times \hat{n}_{s}\right)}\right)^{-2} \hat{\mathbf{\Lambda}}_{s} \hat{\mathbf{E}}_{s}^{\dagger} \mathbf{A}(\mathbf{z}, \mathbf{K})\right)^{-1}
$$

The direct minimization of (40), i.e., the estimation of $\hat{n}_{s}$ optimal elevations $\hat{z}_{i}$ and target vectors $\mathbf{k}_{i}$, requires an optimization over a $5 \hat{n}_{s}$-dimensional space and implies an excessive computational burden. As it has been shown in [5], a reformulation of the NSF criterion (40) permits to efficiently concentrate the optimization with respect to the polarimetric domain and to reduce the search space dimension to that of the SP case. 
Vectors of polarimetric coefficients are defined as

$$
\phi_{p}=\left[k_{1_{p}}, \ldots, k_{\hat{n}_{s_{p}}}\right]^{\mathrm{T}} \in \mathbb{C}^{\hat{n}_{s} \times 1}, \quad p=1,2,3 .
$$

These vectors completely define the polarimetric properties of the $\hat{n}_{s}$ sources since $\mathbf{K}=\left[\boldsymbol{\phi}_{1}, \boldsymbol{\phi}_{2}, \boldsymbol{\phi}_{3}\right]^{\mathrm{T}}$. The polarimetric steering matrix may then be expressed as

$$
\mathbf{A}(\mathbf{z}, \mathbf{K})=\left(\mathbf{I}_{(3 \times 3)} \otimes \mathbf{A}(\mathbf{z})\right) \boldsymbol{\Phi}=\mathbf{B}_{\mathbf{A}}(\mathbf{z}) \boldsymbol{\Phi}
$$

where $\boldsymbol{\Phi} \in \mathbb{C}^{3 \hat{n}_{s} \times \hat{n}_{s}}$ is composed of three diagonal matrices of polarimetric coefficients

$$
\boldsymbol{\Phi}=\left[\boldsymbol{\Phi}_{1} \boldsymbol{\Phi}_{2} \boldsymbol{\Phi}_{3}\right]^{\mathrm{T}}, \quad \text { with } \boldsymbol{\Phi}_{p}=\operatorname{diag}\left(\boldsymbol{\phi}_{p}\right) .
$$

Splitting the augmented steering matrix as $\mathbf{B}_{\mathbf{A}}(\mathbf{z})=$ $\left[\mathbf{A}_{1}(\mathbf{z}) \mathbf{A}_{2}(\mathbf{z}) \mathbf{A}_{3}(\mathbf{z})\right]$, with $\mathbf{A}_{p}(\mathbf{z}) \in \mathbb{C}^{3 \hat{n}_{s} \times \hat{n}_{s}}$, the polarimetric steering matrix may be rewritten as

$$
\mathbf{A}(\mathbf{z}, \mathbf{K})=\mathbf{A}_{1}(\mathbf{z}) \Phi_{1}+\mathbf{A}_{2}(\mathbf{z}) \Phi_{2}+\mathbf{A}_{3}(\mathbf{z}) \Phi_{3} .
$$

Using this alternative expression, the NSF cost function in (40) may be rewritten as

$$
Q_{\mathrm{NSF}}(\mathbf{z}, \mathbf{K})=\sum_{i=1}^{3} \sum_{j=1}^{3} \operatorname{tr}\left(\boldsymbol{\Phi}_{i}^{\dagger} \mathbf{A}_{i}^{\dagger}(\mathbf{z}) \boldsymbol{\Pi} \mathbf{A}_{j}(\mathbf{z}) \boldsymbol{\Phi}_{i} \mathbf{W}\right)
$$

where $\boldsymbol{\Pi}=\hat{\mathbf{E}}_{n} \hat{\mathbf{E}}_{n}^{\dagger}$. Using the properties of the Hadamard product, the FP-NSF cost function may be expressed as a simple quadratic form

$$
Q(\mathbf{z}, \phi)=\phi^{\dagger} \mathbf{M}(\mathbf{z}) \phi
$$

where $\phi=\left[\phi_{1}^{\mathrm{T}}, \boldsymbol{\phi}_{2}^{\mathrm{T}}, \boldsymbol{\phi}_{3}^{\mathrm{T}}\right]^{\mathrm{T}} \in \mathbb{C}^{3 \hat{n}_{s} \times 1}$ represents the polarimetric information, whereas $\mathbf{M}(\mathbf{z}) \in \mathbb{C}^{3 \hat{n}_{s} \times 3 \hat{n}_{s}}$ is composed of $\left(\hat{n}_{s} \times \hat{n}_{s}\right)$ blocks given by $\mathbf{M}_{i j}=\mathbf{A}_{i}^{\dagger}(\mathbf{z}, \mathbf{K}) \boldsymbol{\Pi} \mathbf{A}_{j}(\mathbf{z}, \mathbf{K}) \odot \mathbf{W}$, $i, j=1,2$, or 3 . Despite its very convenient form, the FPNSF cost function in (47) cannot be minimized using an eigendecomposition of $\mathbf{M}(\mathbf{z})$, due to the inherent property of the solution eigenvector, $\hat{\phi}^{\dagger} \hat{\phi}=1$, which may not satisfy the unitary constraint on the scattering vectors $\mathbf{k}_{i}^{\dagger} \mathbf{k}_{i}=1$. Instead, we use a solution, proposed in [8] in the dual-polarization case, based on the transformation of the minimization of (47) into an unconstrained problem, by normalizing $\phi$ by the polarimetric response of the first polarimetric channel, i.e., $\phi^{\prime}=\left[\mathbf{1}_{\left(\hat{n}_{s} \times 1\right)}, \boldsymbol{\phi}_{2}^{\prime T}, \boldsymbol{\phi}_{3}^{\prime T}\right]^{\mathrm{T}}$, where $\left[\boldsymbol{\phi}_{p}^{\prime}\right]_{i}=\left[\boldsymbol{\phi}_{p}\right]_{i} /\left[\boldsymbol{\phi}_{1}\right]_{i}$. If $\boldsymbol{\phi}_{1}$ contains some zero values, a permutation between the different polarimetric channels can easily be performed when forming $\phi$ and reverted once the optimal solutions are found. The FP-NSF criterion becomes $Q\left(\mathbf{z}, \phi^{\prime}\right)=\phi^{\prime \dagger} \mathbf{M}(\mathbf{z}) \phi^{\prime}$.

Solving $\partial Q\left(\mathbf{z}, \boldsymbol{\phi}^{\prime}\right) / \partial \boldsymbol{\phi}_{2}^{\prime}=\partial Q\left(\mathbf{z}, \boldsymbol{\phi}^{\prime}\right) / \partial \boldsymbol{\phi}_{3}^{\prime}=0$ yields

$$
\begin{aligned}
& \hat{\mathbf{\Phi}}_{2}^{\prime}(\mathbf{z})=-\mathbf{M}_{22}^{-1}\left(\mathbf{M}_{21}+\mathbf{M}_{23} \mathbf{G}\right) \mathbf{1}_{\left(\hat{n}_{s} \times 1\right)} \\
& \hat{\mathbf{\Phi}}_{3}^{\prime}(\mathbf{z})=\mathbf{G} \mathbf{1}_{\left(\hat{n}_{s} \times 1\right)}
\end{aligned}
$$

with

$$
\mathbf{G}=\left(\mathbf{M}_{23}^{\dagger} \mathbf{M}_{22}^{-1} \mathbf{M}_{23}-\mathbf{M}_{33}\right)^{-1}\left(\mathbf{M}_{31}-\mathbf{M}_{32} \mathbf{M}_{22}^{-1} \mathbf{M}_{21}\right) \text {. }
$$

Once the optimal polarization configuration $\hat{\phi}$ is found, the FP-NSF cost function depends only on $\mathbf{z}$, and its minimization may be formulated as

$$
\hat{\mathbf{z}}=\arg \min _{\mathbf{z}} Q\left(\mathbf{z}, \hat{\phi}^{\prime}\right)=\arg \min _{\mathbf{z}} \hat{\phi}^{\prime \dagger} \mathbf{M}(\mathbf{z}) \hat{\boldsymbol{\phi}}^{\prime}
$$

whose search dimension is reduced to $\hat{n}_{s}$ like in the SP case. Once the FP-NSF estimator, the position in elevation, and the scattering mechanism of each scatterer are determined, the corresponding reflectivities can be easily obtained by using a polarimetric LS solution.

\section{NUMERICAL EXAMPLES}

In order to demonstrate the performance of the aforementioned tomographic estimators, signals are simulated for two scatterers located at $h_{1}=0 \mathrm{~m}$ and $h_{2}=4 \mathrm{~m}$, with $M=5$ acquisitions. The baselines are assumed to be evenly distributed, and between each successive acquisition, the difference of vertical wavenumbers is $\Delta k_{z}=0.1$. Assuming equal reflectivities, the covariance matrix of source signals is given by

$$
\mathbf{R}_{\mathbf{x}}=\left[\begin{array}{cc}
1 & \rho \\
\rho^{*} & 1
\end{array}\right]
$$

and $S N R=20 \mathrm{~dB}$. By adjusting the correlation factor value $\rho$, $\mathbf{R}_{\mathbf{x}}$ can represent the source covariance matrix of coherent scatterer $(\rho=1)$, distributed scatterer $(\rho=0)$, and hybrid scatterer $(0<\rho<1)$.

\section{A. MB-InSAR Configuration}

First, we fix $\rho=0$ and simulate the sample data covariance matrix with different numbers of looks, in order to investigate the asymptotic properties of the Capon, MUSIC, NSF, and SSF estimators. With the estimation performance for both sources being equal, the analysis concentrates on $h_{2}$ only. Fig. 2(a) shows the rapid convergence of the HR estimators to a low root-mean-square error (rmse) as the number of looks increases. Capon rmse converges to a higher value due to a limited vertical resolution. SSF, NSF, and MUSIC estimators are asymptotically equivalent in rmse for large sample size data. In order to study the vertical resolution, these estimators are applied to uncorrelated signals $(\rho=0)$ with varying height difference between the scatterers $\Delta h=\left|h_{1}-h_{2}\right|$. Fig. 2(b) shows that, when $\Delta h<2 \mathrm{~m}$, MUSIC performance degrades significantly due to closely spaced scatterers. SSF and NSF estimators both provide very good resolution with $\Delta h \geq 0.4 \mathrm{~m}$, whereas Capon's method requires $\Delta h>4 \mathrm{~m}$. The rmse of the estimated $h_{2}$ is plotted with respect to the correlation factor $\rho$ in Fig. 2(c) for a 256-look sample data covariance matrix with $\rho$ varying from 0 to 0.99 . The NSF estimator provides the most accurate estimate for uncorrelated or partially correlated signals $(\rho<0.95)$, whereas the SSF estimator copes well with highly correlated signals $(\rho \geq 0.95)$. MUSIC cannot deal with highly correlated sources due to the quasisingularity of $\mathbf{R}_{\mathrm{x}}$. As mentioned in Section II, the ratio of uncorrelated to coherent scattered power $(U C R)$ determines the statistical properties of hybrid signals, since it influences 


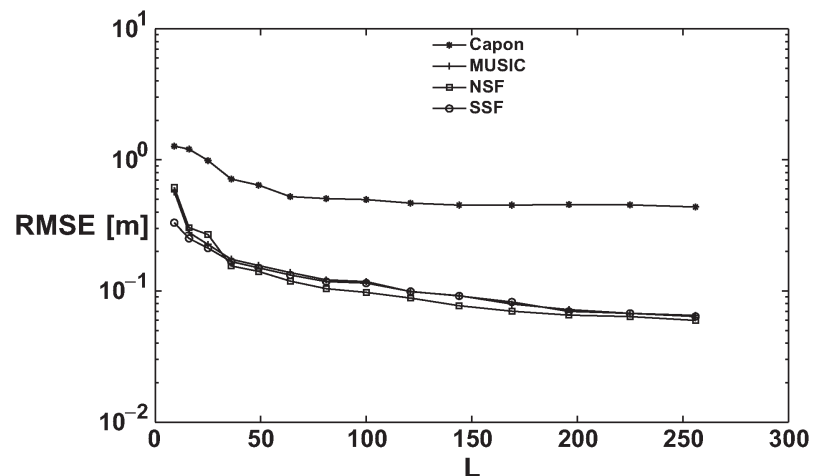

(a)

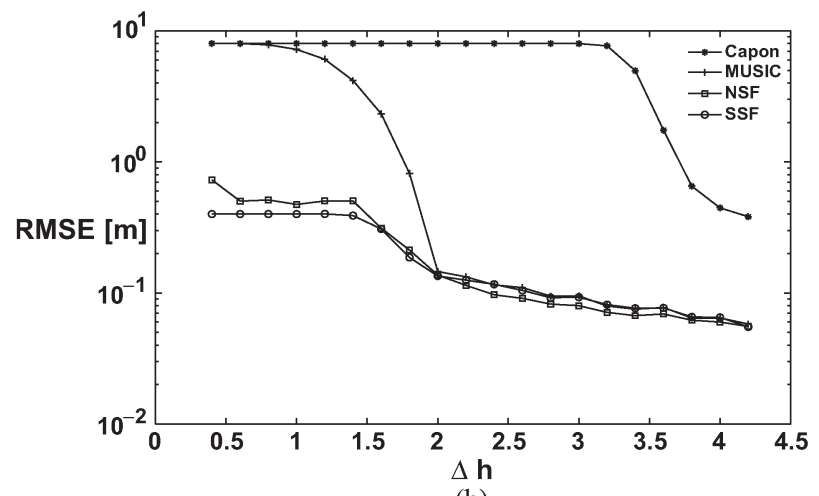

(b)

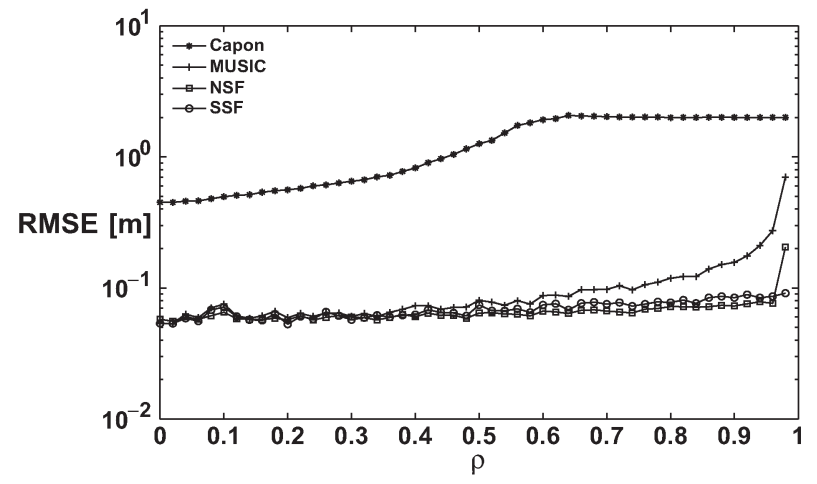

(c)

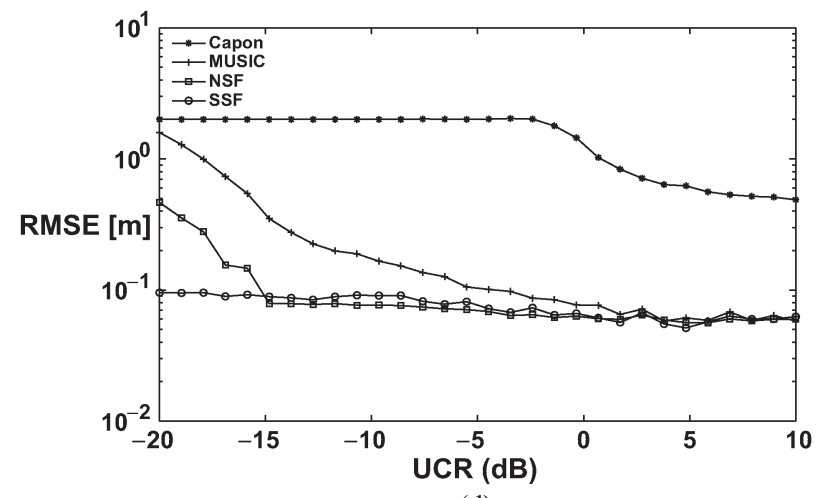

(d)

Fig. 2. MB-InSAR: Height estimation. RMSE of $h$ estimation versus (a) number of looks, (b) height difference when $\rho=0$, (c) source correlation, and (d) $U C R$ (in decibels).

the correlation between sources. A coherent scatterer located at $0 \mathrm{~m}$ and a distributed scatterer at $4 \mathrm{~m}$ with a varying $U C R$ value are simulated. Using the aforementioned estimators, the

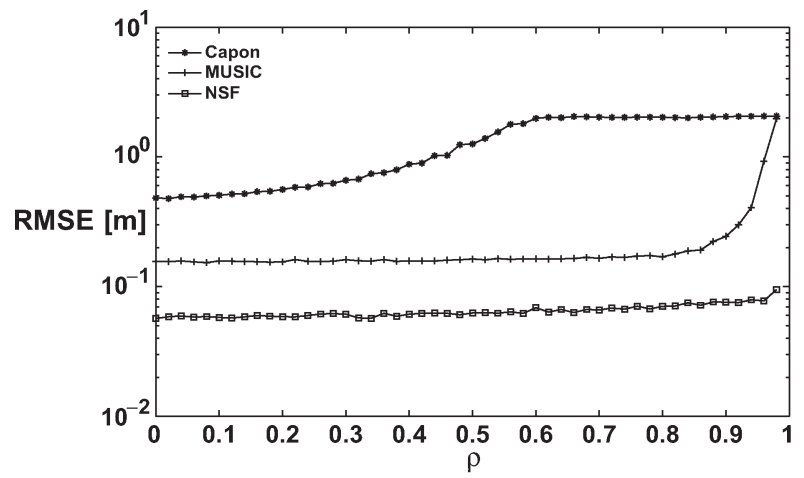

(a)

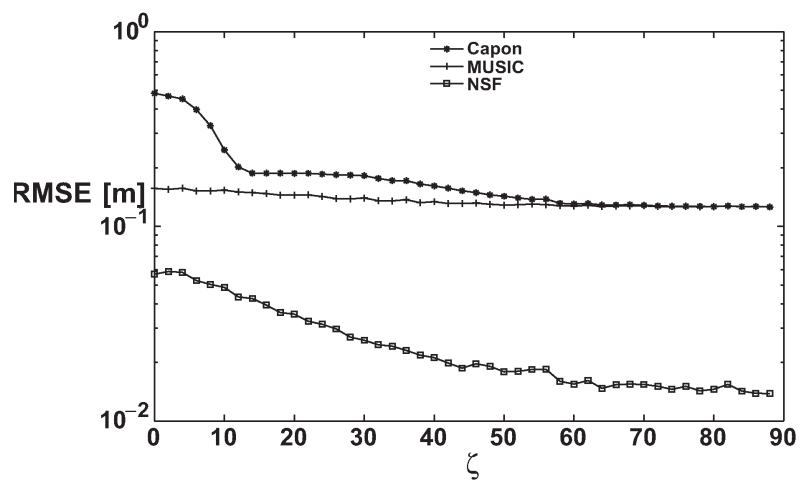

(b)

Fig. 3. MB-PolInSAR height estimation. RMSE of $h$ estimation versus (a) source correlation and (b) polarization difference $\zeta(\rho=0)$.

resulting rmse of $h_{2}$ is shown in Fig. 2(d). Among these estimators, the SSF estimator maintains the best performance when $U C R<-14.5 \mathrm{~dB}$ (i.e., $\rho>0.96$ ), whereas NSF performs best when $U C R \geq-14.5 \mathrm{~dB}$ (i.e., $\rho<0.96$ ). The MUSIC estimator reaches the same estimation accuracy as SSF and NSF estimators when $U C R \geq 3 \mathrm{~dB}$. This fact confirms the results in Fig. 2(c).

\section{B. MB-PolInSAR Configuration}

In an MB-PolInSAR configuration, a 256-look sample covariance matrix is derived from FP signals from two canonical surfacelike scatterers, i.e., $\mathbf{k}_{1}=\mathbf{k}_{2}=[1,0,0]^{\mathrm{T}}$. Fig. 3(a) shows the height estimation rmse w.r.t. $\rho$ : FP-Capon estimator still cannot accurately separate two scatterers due to a limited resolution, FP-NSF estimator provides the most precise height estimate, and FP-MUSIC estimator has a degraded performance for highly correlated scatterers.

The role played by polarization diversity for source separation is investigated, using the polarization difference angle $\zeta$ with $\cos \zeta=\mathbf{k}_{1}^{\dagger} \mathbf{k}_{2} / \sqrt{\left|\mathbf{k}_{1}\right|^{2}\left|\mathbf{k}_{2}\right|^{2}}$. The same surface scattering is maintained for the medium at $0 \mathrm{~m}$, whereas the scattering mechanism at $4 \mathrm{~m}$ varies. As shown in Fig. 3(b), when $\zeta$ increases, the polarization diversity between two scatterers improves the height resolution, particularly for the FP-Capon estimator which reaches the same resolution as that of MUSIC when $\zeta>60^{\circ}$. The FP-NSF estimator performs best over all the polarization difference angles. 


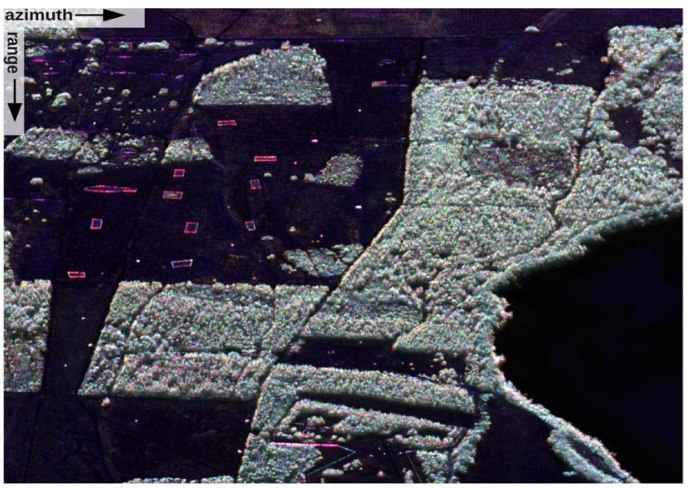

(a)

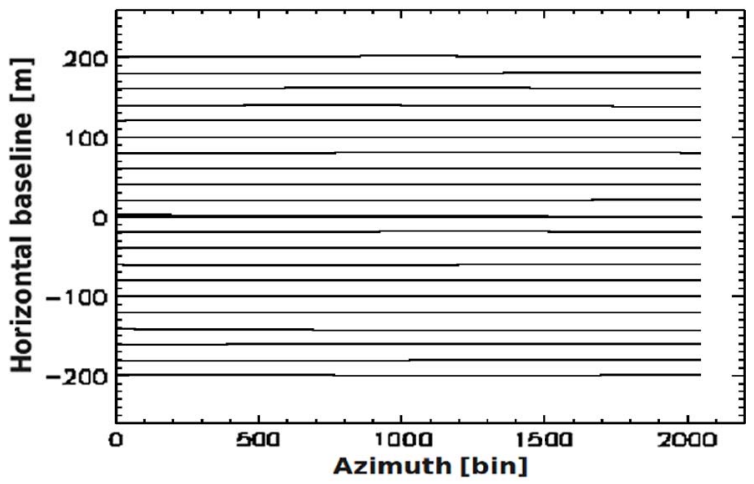

(b)

Fig. 4. Polarimetric SAR image (Pauli basis) of the observed scene and horizontal baseline distribution (courtesy of DLR). (a) Observed scene. (b) Baseline distribution.

\section{EXPERIMENTAL RESULTS}

In this section, the tomographic analysis of a volumetric forested area and subcanopy objects is led with diverse spectral estimators, using FP L-band airborne data acquired by the DLR's E-SAR system over the Dornstetten test site, Germany, shown in Fig. 4(a). The MB-PolInSAR acquisition geometry consists of 23 flight tracks, three of which are located very close to each other (quasi-null spatial baseline) in order to estimate the temporal decorrelation. The horizontal baselines form a quasi-uniform linear array with an average baseline close to 20 m, as shown in Fig. 4(b), and the geometric vertical resolution is about $2 \mathrm{~m}$. This quasi-regular distribution provides 3-D features with reduced sidelobe levels and hence facilitates the tomographic analysis.

\section{A. $M O S$}

Parametric spectral estimators require to estimate the dimension of the signal space, i.e., the number of effective sources present in each resolution cell. The MOS technique developed in [21] is used in this study to estimate this dimension from the eigendecomposition of the data covariance matrix. For the MOS of multicomponent InSAR signals, the techniques based on ITCs have been applied in [11], and a technique based on Capon and LS methods was also developed in [29]. In order to increase the robustness of the ITC methods over speckleaffected scatterers and stabilize the variations of the smallest eigenvalues [30], we resort to the diagonal loading (DL) technique. Unlike single polarimetric MOS techniques that

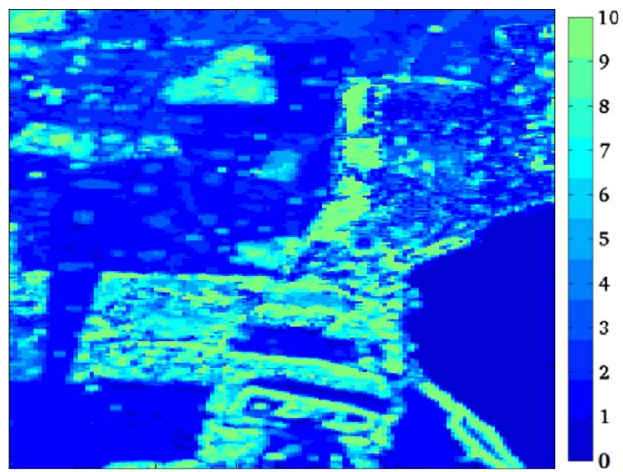

(a)

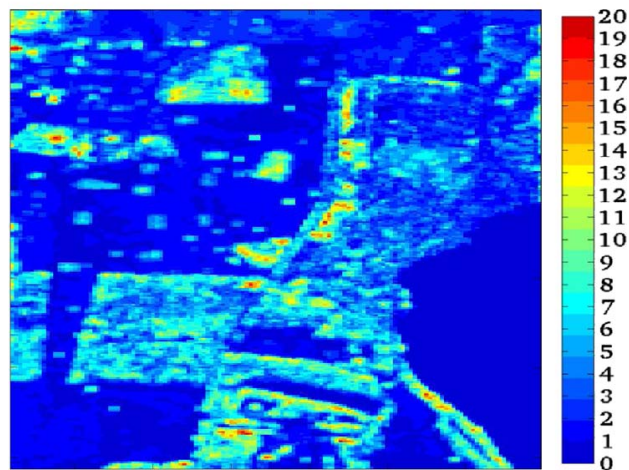

(b)

Fig. 5. MO estimation. (a) DL SP MOS estimation. (b) DL FP MOS estimation.

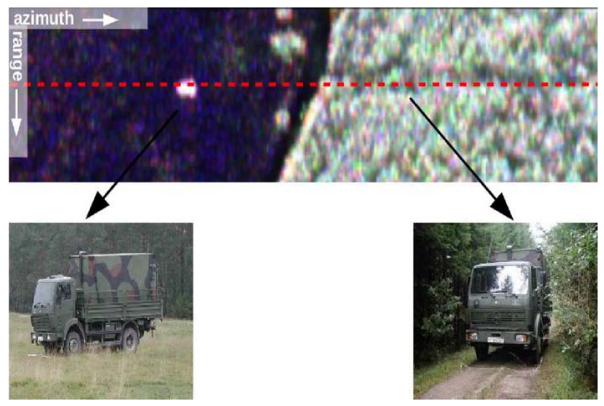

Fig. 6. Setups on the test line.

may overlook sources with a selective polarimetric response, polarimetric MOS techniques use the whole polarimetric space and can detect scatterers with any polarimetric response.

SP and FP MOS techniques with DL and efficient detection criterion 1 criteria are applied to the scene of Dornstetten. The DL value is tuned so that, over bare surfaces, the MO equals one. Fig. 5(b) shows that, using FP MB-InSAR data sets, we obtain a higher MO than with SP ones [Fig. 5(a)]. As it may be seen in Fig. 5, over the lake, the number of estimated sources is equal to zero due to the low backscattered intensity for this kind of smooth medium whose response is dominated by the acquisition noise. Over forested areas, the estimated MO shows intermediate values and reaches a maximum when an underlying object is present in the pixel under consideration.

\section{B. SP Tomograms}

The test zone isolated in Fig. 6 contains under-foliage objects and has been studied in [2] using SP tomograms. The forest 


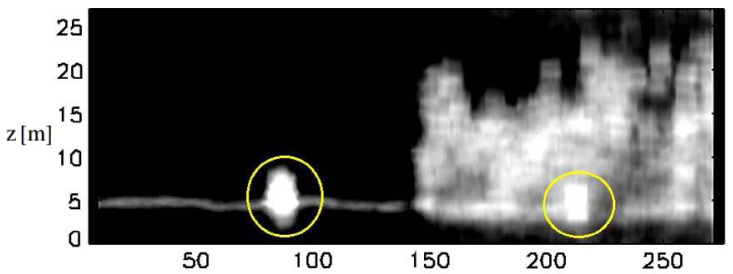

(a)

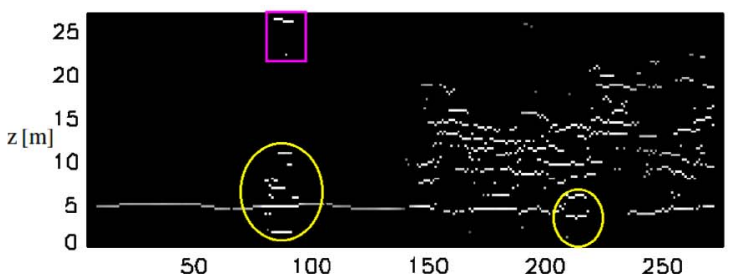

(c)

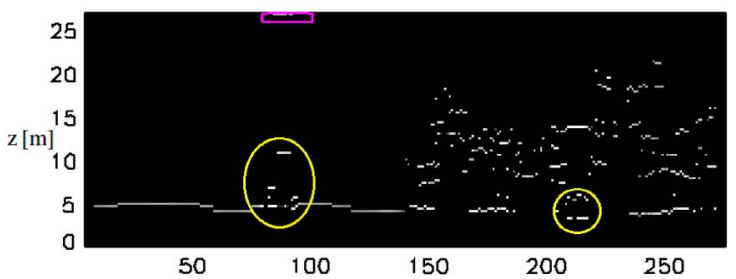

(e)

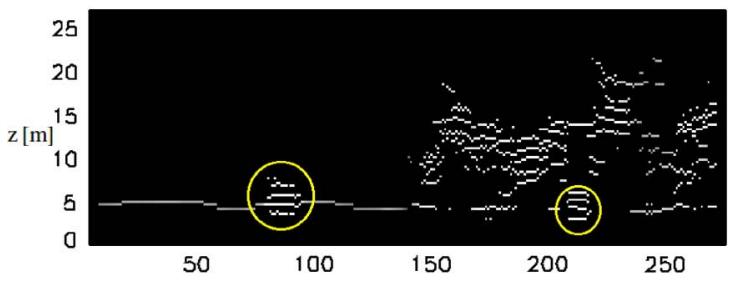

(g)

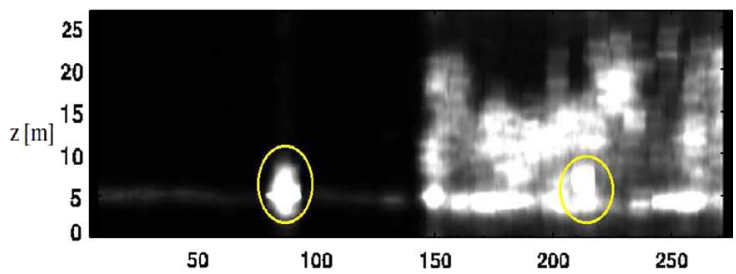

(b)

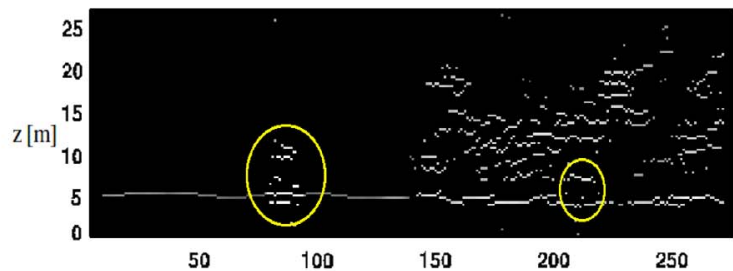

(d)

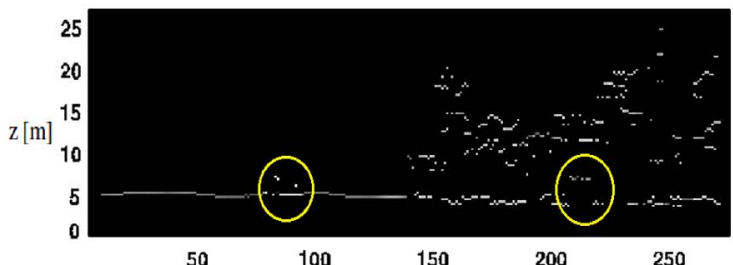

(f)

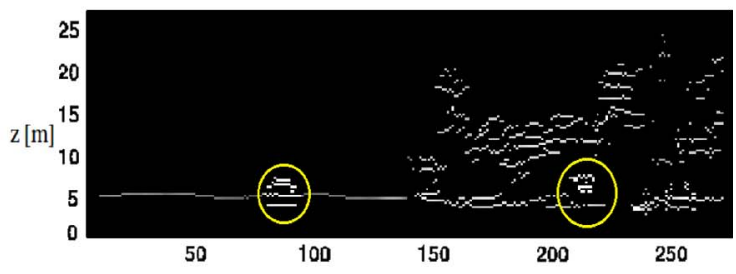

(h)

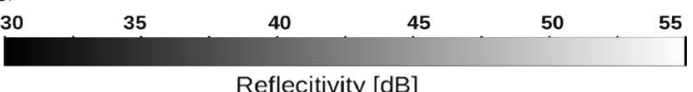

Reflecitivity [dB]

Fig. 7. Reflectivity tomograms. (Yellow circle) Truck. (Violet square) Sidelobe. (Vertical axis) Height (in meters). (Horizontal axis) Azimuth (in bins). A modified color scale is used in Capon spectrum for display purposes. (a) VV-Capon. (b) HH-Capon. (c) VV-MUSIC. (d) HH-MUSIC. (e) VV-NSF. (f) HH-NSF. (g) VV-SSF. (h) HH-SSF.

profile and truck shape were both extracted using Capon's spectral estimation approach. However, as it is well known that nonparametric estimators have limited spectral resolution and sidelobe suppression, particularly in the SP case, we resort to parametric estimators in order to improve the vertical resolution, particularly for objects with complex structures.

Taking a cut in the azimuth direction as in Fig. 6, SP tomograms are computed for the VV channel. The Capon tomogram shown in Fig. 7(a) represents the estimated reflectivity and permits to visualize the forest profile as well as two trucks (yellow circles), one over a bare soil and the other under the forest canopy. The parametric methods MUSIC, NSF, and SSF are applied using the MO shown in Fig. 5(a). The estimated reflectivities vary from 30 to $55 \mathrm{~dB}$. Unlike continuous tomographic approaches, like beamforming and Capon method, parametric techniques provide discrete tomograms with a finite number of sources, as it may be observed in Fig. 7(c), (e), and (g). The MUSIC reflectivity tomogram in Fig. 7(c) enhances the response of the artificial objects but is affected by spurious sidelobes (violet squares), particularly at the location of trucks, due to the fact that MUSIC cannot accurately localize scatterers with highly correlated responses. The NSF and SSF reflectivity tomograms in Fig. 7(e) and $(\mathrm{g})$ perform better than that of MUSIC in terms of sidelobe reduction and height estimation accuracy. The NSF can estimate accurately the height of the truck located beneath the foliage, whose response contains a mixture of distributed (vegetation) and deterministic (truck) contributions, but its performance degrades for the truck over the bare soil whose characteristics are highly coherent. Due to its ability to discriminate coherent scatterers, the SSF method provides an accurate estimate of the uncovered truck, equal to $3 \mathrm{~m}$.

These tomographic estimators are applied to the $\mathrm{HH}$ data sets, and the resulting tomograms are shown in Fig. 7 with reflectivities scaled from 30 to $55 \mathrm{~dB}$. Compared with VV tomograms, scattering from bare soils is weak, but scattering from the top of the under-foliage truck as well as from the underlying ground becomes more energetic. Among these tomograms, the SSF estimator maintains the best estimation for both the truck height and the vertical profile of forests. 


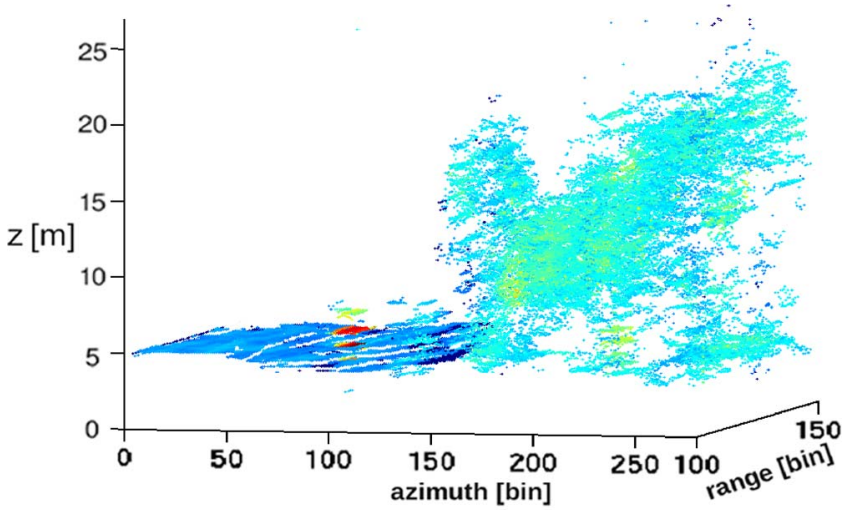

(a)

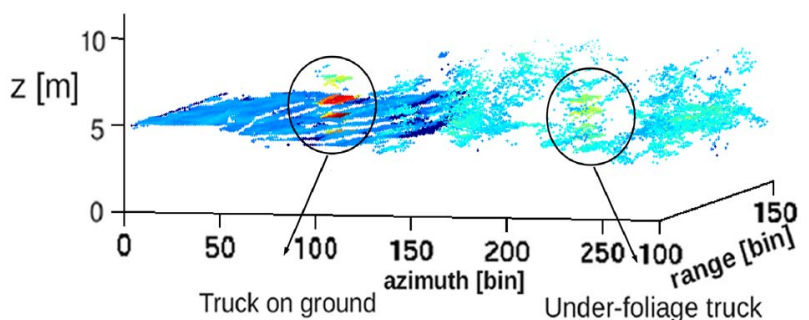

(b)

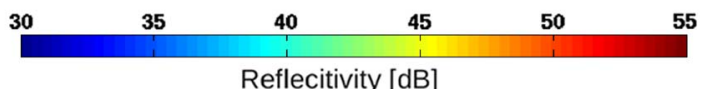

Reflecitivity [dB]

Fig. 8. SP-SSF 3-D reflectivity reconstruction. (a) 3-D reconstruction. (b) 3-D reconstruction with height limitation.

The 3-D tomogram of the area, performed over azimuth bins and for varying range positions using the SSF approach, is shown in Fig. 8(a). The forest canopy and the truck profile over the bare soil can clearly be discriminated. Limiting the reconstruction height to $4 \mathrm{~m}$ above the terrain [Fig. 8(b)] permits to isolate the under-foliage truck response. Its reconstructed shape is similar to the uncovered one, with a reflectivity slightly higher (about $5 \mathrm{~dB}$ more) than the surrounding environment one.

\section{FP Tomograms}

The same azimuth cut is imaged using polarimetric spectral estimators with the MO estimated in Fig. 5(b), and reflectivity tomograms are shown in Fig. 9. Compared with the SP case, the reflectivity tomograms illustrate some improvements due to polarimetric diversity. The Capon tomogram in Fig. 9(a) shows an improved resolution, leading to a better retrieval of vertical profiles for both trucks and forests, but the limited spectral resolution still affects the accuracy of the height estimation over the truck embedded in the forest. The MUSIC tomogram in Fig. 9(b) shows less sidelobes, particularly for the uncovered truck, but still overestimates its height. The NSF estimator in Fig. 9(c) refines the features of the uncovered truck, whose height may be estimated around $3 \mathrm{~m}$.

The tomograms of the polarimetric $\alpha$ angle [18], obtained from the unitary target vector $\mathbf{k}$ estimated by FP estimators, are shown in Fig. 10. For the truck outside the forest, the FPCapon method results in an accurate estimate of the doublebounce reflection at the ground-truck interaction, but its limited vertical resolution results in a single scattering mechanism for

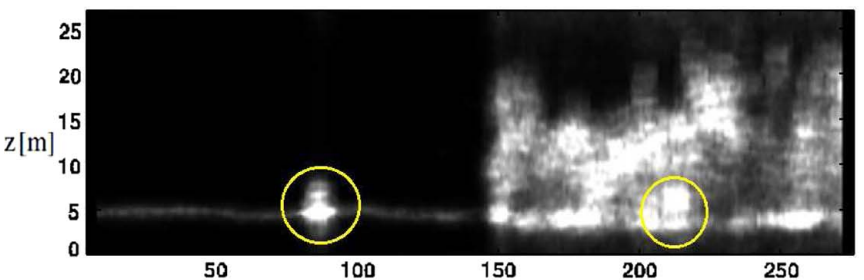

(a)

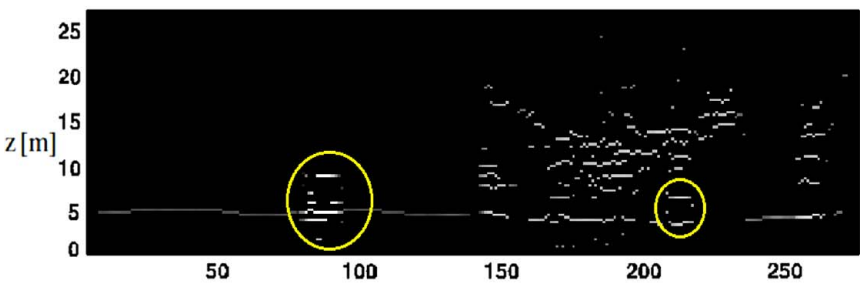

(b)
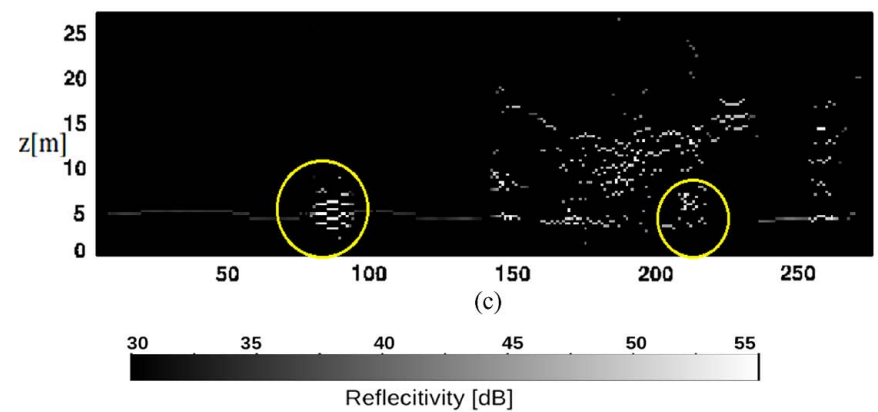

Fig. 9. Reflectivity tomograms. (Circle) Truck. (Vertical axis) Height (in meters). (Horizontal axis) Azimuth (in bins). A modified color scale is used in Capon spectrum for display purposes. (a) FP-Capon. (b) FP-MUSIC. (c) FP-NSF.

the whole truck, as shown in Fig. 10(a). In Fig. 10(b), the FPMUSIC estimator also precisely estimates the ground-truck reflection but suffers from sidelobes, whereas the FP-NSF estimator can precisely estimate scattering mechanisms from the truck with surfacelike scattering mechanisms over the top of the truck and double-bounce reflection on the ground in Fig. 10(c). For the truck beneath the foliage, Capon's method cannot accurately estimate its height, but the estimated scattering mechanism is quite reliable, i.e., double-bounce reflection between truck top and trunk as well as the one at the ground-truck interaction. The MUSIC estimator provides a quite good estimate for both truck height and the associated scattering mechanisms. The FP-NSF estimator provides a good height estimate describing the truck's structure but is sensitive to the degree of polarization. This may be due to the fact that the polarimetric response of the under-foliage truck is affected by the penetration through the canopy as well as by the sidelobes of the volume contribution in the vertical direction. Within the forest canopy, the $\alpha$ value reaches low due to an almost white polarimetric covariance matrix. In this case, the entropy tends to equal one, indicating that the value of $\alpha$ makes no physical sense.

The FP-NSF estimator is applied over the whole test zone, and the 3-D reconstruction of the parameter $\alpha$ value is shown in Fig. 11. Fig. 11(a) shows that the truck outside the forest has a strong double-bounce reflection at the ground-truck interaction and the shape of the truck beneath the canopy is precisely reconstructed with an $\alpha$ value around $45^{\circ}$ due to a mixture of 


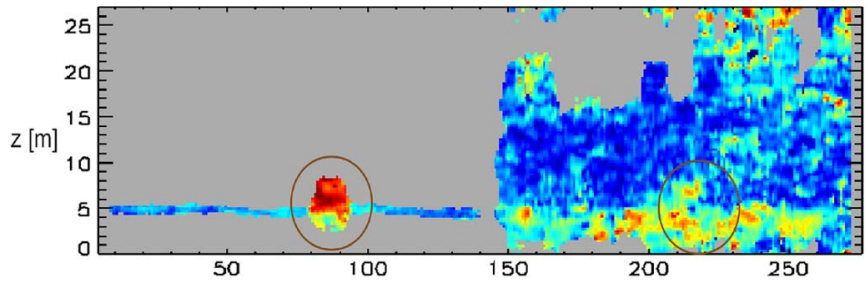

(a)

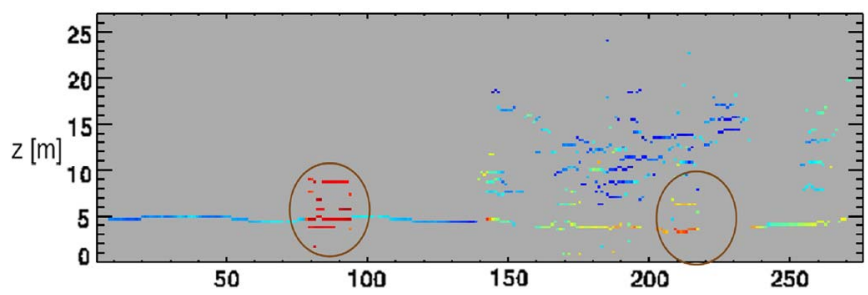

(b)

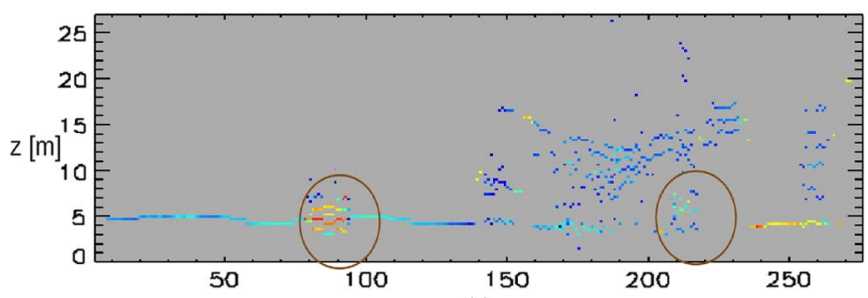

(c)

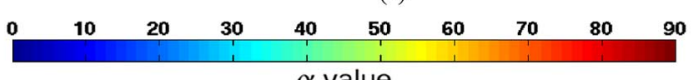

$\alpha$ value

Fig. 10. $\alpha$ tomograms. (Circle) Truck. (Vertical axis) Height (in meters). (Horizontal axis) Azimuth (in bins). (a) FP-Capon. (b) FP-MUSIC. (c) FP-NSF.

multiple reflections, i.e., double-bounce reflection between the truck top and the trunk, volume scattering from the canopy, etc. In order to better visualize the underlying truck response, the height is limited to $4 \mathrm{~m}$ above the ground, and the reflectivity is limited to values inferior to $43 \mathrm{~dB}$ [Fig. 11(b)]. The underfoliage truck is well described in terms of both shape and scattering patterns.

\section{CONCLUSION AND Future WORK}

In this paper, under-foliage object imaging has been investigated using SP and FP SAR tomographies. Several parametric estimators like MUSIC, NSF, and SSF are used to extract the shape of under-foliage objects and the forest profile. Compared to features with a limited resolution shown by Capon tomograms, parametric estimators provide a better vertical resolution for localizing objects with complex structures, whereas they may be conditioned by their statistical behaviors. The MUSIC performance degrades due to highly correlated scatterers and results in many spurious sidelobes. The NSF estimator outperforms MUSIC in terms of sidelobe reduction but still cannot cope with coherent scatterers like the truck over the bare soil. The model-adaptive estimator SSF, dealing with distributed and coherent scatterers, gives a precise estimation for the profiles of trucks and forests.

Compared to SP SAR tomography, POLTOMSAR techniques permit to extract some more physical features. Subcanopy objects can be more reliably characterized by scattering mechanisms obtained by POLTOMSAR, in addition to the shape and reflectivity provided by SP tomography. The

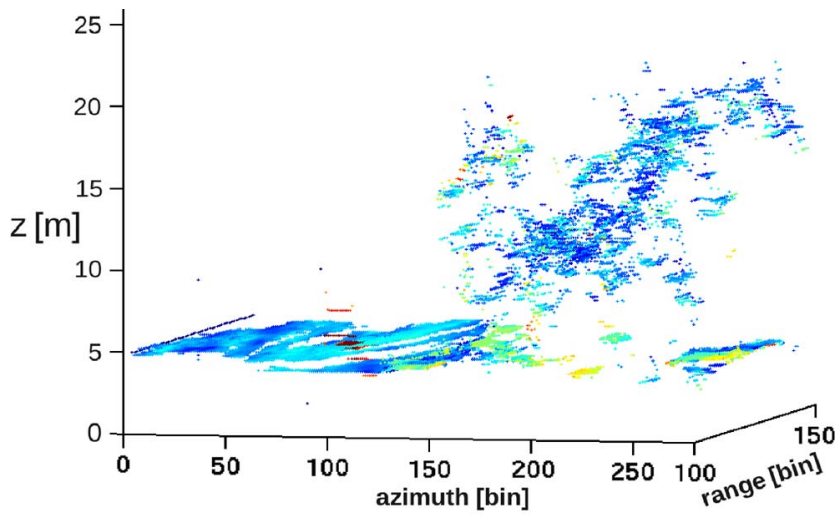

(a)

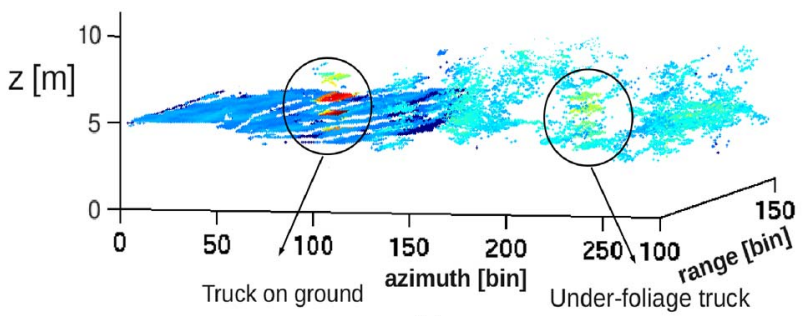

(b)

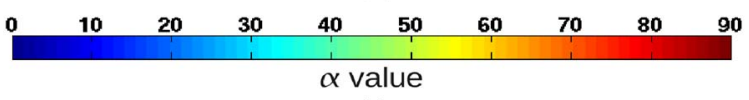

(c)

Fig. 11. FP-NSF 3-D $\alpha$ reconstruction. (a) 3-D reconstruction. (b) 3-D reconstruction with height and reflectivity limitations.

proposed PF-NSF estimator outperforms other polarimetry estimators for localizing deterministic scatterers and refining the estimation of their scattering mechanisms.

By means of 3-D reconstruction over the test zone, the forest and the uncovered truck are both precisely visualized. As to the under-foliage truck, SP tomographic reconstruction by SSF correctly reconstructs the truck shape with a reflectivity slightly higher compared to the associated environment one. Using FPNSF tomographic reconstruction, one can clearly distinguish the underlying truck by means of scattering mechanisms.

\section{ACKNOWLEDGMENT}

This study is based on data acquired in the frame of the SAR tomography project, funded by eOsphere Ltd. and EMRS DTC U.K. The authors would like to thank the Experimental Synthetic Aperture Radar system team of the German Aerospace Center (DLR) for their efforts in conducting and processing this campaign and Dr. M. Nannini for kindly providing ground measurement information.

\section{REFERENCES}

[1] A. Reigber and A. Moreira, "First demonstration of airborne SAR tomography using multibaseline L-band data," IEEE Trans. Geosci. Remote Sens., vol. 38, no. 5, pp. 2142-2152, Sep. 2000.

[2] M. Nannini, R. Scheiber, and R. Horn, "Imaging of targets beneath foliage with SAR tomography," in Proc. EUSAR, Jun. 2008, pp. 1-4.

[3] S. Tebaldini, "Algebraic synthesis of forest scenarios from multibaseline PolInSAR data," IEEE Trans. Geosci. Remote Sens., vol. 47, no. 12, pp. 4132-4142, Dec. 2009.

[4] P. Stoica and A. Nehorai, "Performance study of conditional and unconditional direction-of-arrival estimation," IEEE Trans. Acoust., Speech, Signal Process., vol. 38, no. 10, pp. 1783-1795, Oct. 1990. 
[5] Y. Huang and L. Ferro-Famil, "Building height estimation using multibaseline L-band SAR data and polarimetric weighted subspace fitting methods," in Proc. PolInSAR, Frascatti, Jan. 2009.

[6] P. Stoica and K. Sharman, "Maximum likelihood methods for directionof-arrival estimation," IEEE Trans. Acoust., Speech, Signal Process., vol. 38, no. 7, pp. 1132-1143, Jul. 1990.

[7] M. Viberg and B. Ottersten, "Sensor array processing based on subspace fitting," IEEE Trans. Signal Process., vol. 39, no. 5, pp. 1110-1121, May 1991.

[8] A. Swindlehurst and M. Viberg, "Subspace fitting with diversely polarized antenna arrays," IEEE Trans. Antennas Propag., vol. 41, no. 12, pp. 16871694, Dec. 1993.

[9] F. Gini and F. Lombardini, "Multibaseline cross-track SAR interferometry: A signal processing perspective," IEEE Aerosp. Electron. Syst. Mag., vol. 20, no. 8, pp. 71-93, Aug. 2005.

[10] F. Gini, F. Lombardini, and M. Montanari, "Layover solution in multibaseline SAR interferometry," IEEE Trans. Aerosp. Electron. Syst., vol. 38, no. 4, pp. 1344-1356, Oct. 2002.

[11] F. Gini and F. Bordoni, "On the behavior of information theoretic criteria for model order selection of InSAR signals corrupted by multiplicative noise," Signal Process., vol. 83, no. 5, pp. 1047-1063, May 2003.

[12] L. Ferro-Famil, A. Reigber, and E. Pottier, "Nonstationary natural media analysis from polarimetric SAR data using a two-dimensional time-frequency decomposition approach," Can. J. Remote Sens., vol. 31, no. 1, pp. 21-29, 2005.

[13] L. Ferro-Famil and E. Pottier, "Urban area remote sensing from L-band PolSAR data using time-frequency techniques," in Proc. IEEE Urban Remote Sens. Joint Event, Apr. 2007, pp. 5045-5048.

[14] S. Sauer, L. Ferro-Famil, E. Pottier, and A. Reigber, "Physical parameter extraction over urban areas using L-band PolSAR data and interferometric baseline diversity," in Proc. IEEE IGARSS, Jul. 2007, pp. 1-5.

[15] S. Sauer, L. Ferro-Famil, A. Reigber, and E. Pottier, "Three-dimensional imaging and scattering mechanism estimation over urban scenes using dual-baseline polarimetric insar observations at 1-band," IEEE Trans. Geosci. Remote Sens., vol. 49, no. 11, pp. 4616-4629, Jun. 2011.

[16] P. Stoica and A. Nehorai, "MUSIC, maximum likelihood, and Cramer-Rao bound," IEEE Trans. Acoust., Speech, Signal Process., vol. 37, no. 5, pp. 702-741, May 1989.

[17] P. Stoica and A. Nehorai, "MUSIC, maximum likelihood, and Cramer-Rao bound: Further results and comparisons," IEEE Trans. Acoust., Speech, Signal Process., vol. 38, no. 12, pp. 2140-2150, Dec. 1990.

[18] S. R. Cloude and E. Pottier, "A review of target decomposition theorems in radar polarimetry," IEEE Trans. Geosci. Remote Sens., vol. 34, no. 2, pp. 498-518, Mar. 1996.

[19] K. J. Sangston, F. Gini, M. V. Greco, and A. Farina, "Structures for radar detection in compound Gaussian clutter," IEEE Trans. Aerosp. Electron. Syst., vol. 35, no. 2, pp. 445-458, Apr. 1999.

[20] F. Gini and M. Greco, "Covariance matrix estimation for CFAR detection in correlated heavy tailed clutter," Signal Process., vol. 82, no. 12, pp. 1847-1859, Dec. 2002.

[21] M. Wax and T. Kailath, "Detection of signals by information theoretic criteria," IEEE Trans. Acoust., Speech, Signal Process., vol. ASSP-33, no. 2, pp. 387-392, Apr. 1985.

[22] M. Wax and I. Ziskind, "Detection of the number of coherent signals by the MDL principle," IEEE Trans. Acoust., Speech, Signal Process., vol. 37, no. 8, pp. 1190-1196, Aug. 1989.

[23] M. Wax and I. Ziskind, "Detection and localization of multiple sources via the stochastic signal model," IEEE Trans. Signal Process., vol. 39, no. 11, pp. 2450-2456, Nov. 1991.

[24] M. G. Christensen, A. Jakobsson, and S. H. Jensen, "Sinusoidal order estimation using the subspace orthogonality and shift-invariance properties," in Conf. Rec. Asilomar Conf. Signals, Syst., Comput., 2007, pp. 651-655.

[25] M. G. Christensen, A. Jakobsson, and S. H. Jensen, "Joint high-resolution fundamental frequency and order estimation," IEEE Trans. Audio, Speech, Lang. Process., vol. 15, no. 5, pp. 1635-1644, Jul. 2007.

[26] P. Stoica and R. Moses, Spectral Analysis of Signals. Englewood Cliffs, NJ: Prentice-Hall, 2005.

[27] B. Ottersten, M. Viberg, P. Stoica, and A. Nehorai, "Exact and large sample ML techniques for parameter estimation and detection in array processing," in Radar Array Processing. Berlin, Germany: SpringerVerlag, 1993, pp. 99-151.

[28] Y. Huang and L. Ferro-Famil, "3D characterization of buildings in a dense urban environment using L-band PolInSAR data with irregular baselines," in Proc. IGARSS, Jul. 2009, pp. III-29-III-32.
[29] F. Bordoni, F. Gini, and L. Verrazzani, "Capon-LS for model order selection of multicomponent interferometric SAR signals," Proc. Inst. Elect. Eng.-Radar, Sonar Navig., vol. 151, no. 5, pp. 299-305, Oct. 2004.

[30] F. Lombardini and F. Gini, "Model order selection in multi-baseline interferometric radar systems," EURASIP J. Appl. Signal Process., vol. 2005, no. 20, pp. 3206-3219, 2005.

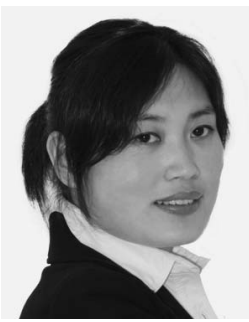

Yue Huang received the M.Sc. degree in electronics, electrical engineering, and automation and systems from University Paul Sabatier, Toulouse, France, in 2007 and the Ph.D. degree in signal processing and telecommunication from the University of Rennes 1, Rennes, France, in 2011, where she was a Ph.D. student from 2007 to 2011 .

She is currently with the SAR Polarimetry, Holography, Interferometry and Radargrammetry Team, Institute of Electronics and Telecommunications of Rennes, University of Rennes 1, where she works in the field of tomographic processing using multibaseline polarimetric and interferometric synthetic aperture radar (SAR) (PolInSAR) data for urban and forestry remote sensing. Her research interests include PolInSAR techniques, SAR interferometry and differential interferometry, physical parameter retrieval, 3-D environment reconstruction, target detection, SAR signal processing, SAR tomography, multivariate spectral estimation methods, and blind source separation.

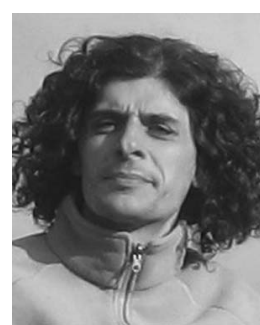

Laurent Ferro-Famil (S'99-A'00-M'04) received the Laurea degree in electronics systems and computer engineering, the M.S. degree in electronics, and the Ph.D. degree from the University of Nantes, Nantes, France, in 1996, 1996, and 2000, respectively.

$\mathrm{He}$ is currently with the University of Rennes 1, Rennes, France, where he was an Associate Professor in 2001, has been a Full Professor since 2011, and is currently the Head of the Radar Polarimetry Remote Sensing Group, Institute of Electronics and Telecommunications of Rennes. His current activities in education are concerned with analog electronics, digital communications, microwave theory, signal processing, and polarimetric synthetic aperture radar (SAR) remote sensing. He is particularly interested in polarimetric SAR signal processing, radar polarimetry theory, and natural media remote sensing using multibaseline polarimetric and interferometric SAR data, with application to classification, electromagnetic scattering modeling and physical parameter retrieval, time-frequency analysis, and 3-D reconstruction of environments.

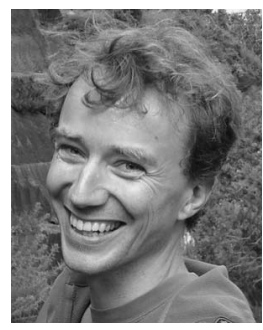

Andreas Reigber (M'02-SM'10) was born in Munich, Germany, in 1970. He received the Diploma degree in physics from the University of Constance, Konstanz, Germany, in 1997, the Ph.D. degree from the University of Stuttgart, Stuttgart, Germany, in 2001, and the Habilitation from Berlin University of Technology, Berlin, Germany, in 2008.

From 1996 to 2000, he was with the Microwave and Radar Institute, German Aerospace Center (DLR), Oberpfaffenhofen, Germany, where he worked in the field of polarimetric synthetic aperture radar (SAR) tomography. In 2001, he was with the Antenna, Radar and Telecom laboratories, University of Rennes 1, Rennes, France, where he was a Postdoctoral Fellow on radar polarimetry and polarimetric interferometry. From 2002 to 2007, he was a Research Associate with the Computer Vision and Remote Sensing laboratories. Since 2008, he has been back with the Microwave and Radar Institute, DLR, where he is currently the Head of the SAR Technology Department and directing the airborne SAR activities of the institute. His current main research interests include the various aspects of multimodal SAR, like SAR interferometry, SAR polarimetry, SAR tomography, and time-frequency analyses, as well as filtering and classification aspects of high-resolution SAR data.

Dr. Reigber was a recipient of the European Conference on Synthetic Aperture Radar 2000 Student Prize Paper Award for an article on SAR remote sensing of forests, the IEEE TRANSACTIONS ON GEOSCIENCE AND REMOTE SENSING (TGRS) Prize Paper Award in 2001 for a work on polarimetric SAR tomography, and the IEEE TGRS Letters Prize Paper Award in 2006 for a work on multipass SAR processing. 